\title{
FDG-PET/CT imaging findings of hepatic tumors and tumor-like lesions based on molecular background
}

\author{
Kumi Ozaki ${ }^{1} \cdot$ Kenichi Harada $^{2} \cdot$ Noboru Terayama $^{3} \cdot$ Nobuyuki Kosaka $^{1} \cdot$ Hirohiko Kimura $^{1} \cdot$ Toshifumi Gabata $^{4}$
}

Received: 20 January 2020 / Accepted: 24 March 2020 / Published online: 3 April 2020

(C) Japan Radiological Society 2020

\begin{abstract}
The usefulness of whole-body 18-fluoro-2-deoxyglucose (FDG)-fluorodeoxyglucose positron emission (PET)/computed tomography (CT) is established for assessment of disease staging, detection of early disease recurrence, therapeutic evaluation, and predicting prognosis in various malignancies; and for evaluating the spread of inflammation. However, the role of FDG-PET/CT for the liver is limited because CT and magnetic resonance imaging (MRI) can provide an accurate diagnosis of most tumors. In addition, in other potentially useful roles there are several pitfalls in the interpretation of FDG uptake in PET/CT imaging. Accurate evaluation demands knowledge of the FDG uptake of each lesion, including potential negative and positive uptakes, and requires an understanding of the underlying background of the molecular mechanisms. The degree of FDG uptake is dependent on cellular metabolic rate and the expression of glucose transporter, hexokinase, and glucose-6-phosphatase, which in turn are closely affected by biological characteristics such as pathological category (e.g., adenocarcinoma, squamous cell carcinoma, small cell cancer, transitional cell cancer, neuroendocrine tumor, sarcoma, lymphoma), tumor differentiation, histological behavior (e.g., solid, cystic, mucinous), and intratumoral alterations (e.g., necrosis, degeneration, hemorrhage). Correlation with the CT and MRI findings, which also precisely depict the pathological findings, is important to avoid misdiagnosis.
\end{abstract}

Keywords Fluorodeoxyglucose $\cdot$ Positron emission/computed tomography $\cdot$ Hepatic tumors $\cdot$ Glucose transporter . Glucose-6-phosphatase complex

\section{Introduction}

18-Fluoro-2-deoxyglucose (FDG)-positron emission tomography (PET)/computed tomography (CT) is a clinical molecular imaging modality that elucidates cellular glucose metabolism. The utility of whole-body FDG-PET/CT is established for the assessment of disease staging, detection of early disease recurrence, evaluation of therapeutic

Kumi Ozaki

ozakik-rad@umin.org

1 Department of Radiology, Faculty of Medical Sciences, University of Fukui, 23-3 Matsuoka-Shimoaizuki, Eiheiji, Fukui 910-1193, Japan

2 Department of Pathology, Kanazawa University Graduate School of Medicine, Kanazawa, Japan

3 Department of Radiology, Takaoka City Hospital, Takaoka, Japan

4 Department of Radiology, Kanazawa University Graduate School of Medicine, Kanazawa, Japan response, and prediction of prognosis in various malignancies, and for evaluating the spread of inflammatory disease. $\mathrm{CT}$ and magnetic resonance imaging (MRI) alone can accurately diagnose a wide range of hepatic tumors and tumorlike lesions. Although the role of FDG-PET/CT is limited in this regard, it nevertheless appears to have certain potentially useful roles.

The majority of primary and secondary malignant hepatic tumors show high FDG uptake, whereas most benign lesions have low uptake or that similar to background liver. However, false-negative and false-positive uptake can occur in such as low uptake in malignant tumors, and high uptake in benign tumors and in inflammatory and infectious lesions. Furthermore, the degree of uptake can differ even among histologically identical tumors, depending on tumor differentiation; and in certain tumors, the degree of uptake can predict prognosis and therapeutic response. Because various factors affect FDG uptake, accurate evaluation of FDG-PET/ $\mathrm{CT}$ imaging requires a sound understanding of the underlying molecular background. 
Table 1 Hepatic tumors and tumor-like lesions

\begin{tabular}{|c|c|}
\hline Malignant tumor & $\begin{array}{l}\text { Benign tumor and tumor-like } \\
\text { lesion }\end{array}$ \\
\hline Hepatocellular carcinoma & Hepatocellular adenoma \\
\hline Intrahepatic cholangiocarcinoma & $\begin{array}{l}\text { Hepatic cavernous hemangioma } \\
\text { and sclerosed hemangioma }\end{array}$ \\
\hline $\begin{array}{l}\text { Combined hepatocellular cholan- } \\
\text { giocarcinoma }\end{array}$ & Focal nodular hyperplasia \\
\hline Hepatic angiosarcoma & Angiomyolipoma \\
\hline Malignant lymphoma & Inflammatory pseudotumor \\
\hline Neuroendocrine tumor & Pseudolymphoma \\
\hline \multirow[t]{3}{*}{ Hepatic metastasis } & Inflammation and infection \\
\hline & $\begin{array}{l}\text { Focal sinusoidal obstruction } \\
\text { syndrome }\end{array}$ \\
\hline & $\begin{array}{l}\text { Intratumoral hemorrhage in acute } \\
\text { phase }\end{array}$ \\
\hline
\end{tabular}

The aim of this article is to review the FDG-PET/CT imaging features of various hepatic tumors and tumor-like lesions (Table 1), and to assess the clinical significance of FDG-PET/CT imaging in the diagnosis, tumor differentiation, and prognosis.

\section{Mechanism of FDG uptake in hepatocytes and influencing factors}

FDG, an analog of glucose that enables evaluation of glucose metabolism, is the most commonly used PET tracer. FDG is taken up into cells by glucose transporters (GLUT) and phosphorylated by hexokinase (HK) to FDG-6-phosphate. FDG-6-phosphate is not a substrate for glycolysis and does not undergo further metabolism, and is dephosphorylated by glucose-6-phosphatase (G6Pase). Dephosphorylated FDG is transported outside of cells by GLUT to maintain glucose homeostasis [1] (Fig. 1). GLUTs are glycoproteins, of which 12 isoforms have so far been identified in different organs [2]. Four mammalian hexokinase isozymes can occur in a single species, each providing a different function [3]. In normal hepatocytes, FDG or glucose is taken into the cytoplasm by GLUT-2, and is phosphorylated by HK-IV. The specific enzyme G6Pase is found mainly in the liver and the kidneys [4]. It has a complicated construction and is associated with the endoplasmic reticulum as G6Pase Complex, comprising the G6Pase catalytic subunits of glucose-6-phosphate transporter (G6PT)1, G6PT2, and G6PT3, which allow to transport FDG-6-phosphate, FDG, and phosphate, respectively, to cross the membrane of the endoplasmic reticulum [5] (Fig. 1).

In hepatic lesions, the disorder of one or more glycolytic pathways traps FDG-6-phosphate within the cell [6]. The degree of uptake is related to the cellular metabolic rate

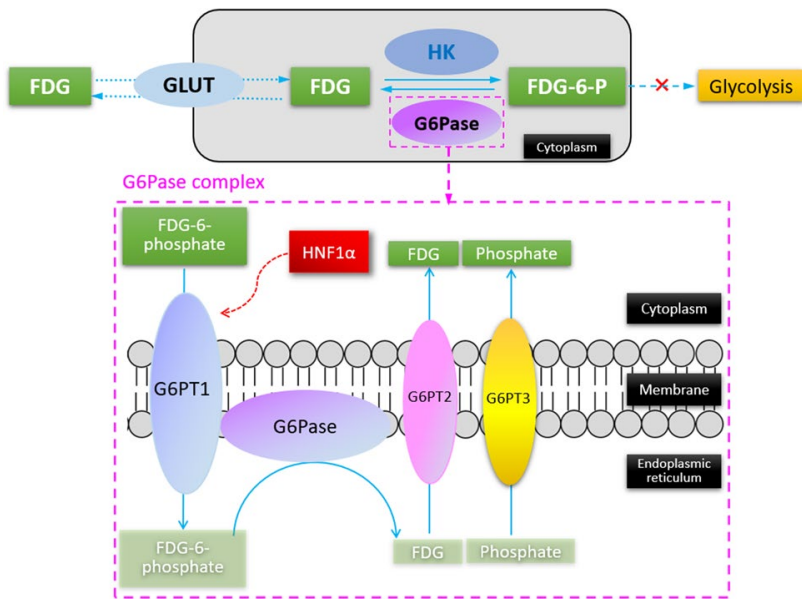

Fig. 1 18-Fluoro-2-deoxyglucose (FDG), an analog of glucose that enables evaluation of glucose metabolism, is the most commonly used PET tracer. FDG is taken up into cells by glucose transporters (GLUT) and phosphorylated by hexokinase (HK) to FDG-6-phosphate. FDG-6-phosphate is not a substrate for glycolysis and does not undergo further metabolism, and is dephosphorylated by glucose6-phosphatase (G6Pase). Dephosphorylated FDG is transported out of the cell by GLUT to maintain glucose homeostasis. GLUT and HK have several isoforms and isozymes, respectively. In normal hepatocytes, FDG or glucose is taken into the cytoplasm by GLUT-2, and is phosphorylated by HK-IV. G6Pase is a specific enzyme found mainly in the liver and the kidneys. G6Pase has a complicated construction and is associated with the endoplasmic reticulum as G6Pase complex. The G6Pase complex comprises G6Pase catalytic subunit glucose6-phosphate transporter (G6PT)1, G6PT2, and G6PT3, which allow to transfer FDG-6-phosphate, FDG, and phosphate, respectively, to cross the endoplasmic reticular membrane. Note that the inactivation of G6PT1 is transcriptionally regulated by hepatocyte nuclear factor (HNF) $1 \alpha$

and the expression of GLUT, HK, and G6Pase [7], which have close relationships with biologic characteristics [8,9], including the pathological category (e.g., adenocarcinoma, squamous cell carcinoma, small cell cancer, transitional cell cancer, neuroendocrine tumor, sarcoma, lymphoma) [10]; histological behavior such as solid, cystic, and mucinous, tumor differentiation; and intratumoral alterations such as necrosis, degeneration, and hemorrhage.

\section{Factors that influence FDG-PET/CT imaging}

Before discussing FDG uptake in various hepatic lesions, it is useful to first consider the potential diagnostic pitfalls in $\mathrm{PET} / \mathrm{CT}$ imaging.

\section{Technical pitfalls}

The potential technical pitfalls in PET/CT imaging include misregistration, truncation, attenuation correction, and partial volume effect. 
Anatomic malalignment of the PET and CT datasets is termed PET/CT misregistration. Incorrect superimposition of FDG activity onto the anatomic structures demonstrated in the CT component [11] can result in a false-negative assessment. Misregistration can be differences in any of respiratory motion, patient motion, and bowel motility between the CT and PET acquisitions [12].

Truncation artifacts on PET/CT are caused by differences in size between the CT $(50 \mathrm{~cm})$ and PET $(70 \mathrm{~cm})$ fields of view, and are seen most frequently in large patients and in patients scanned with the arms down. When the patient's body extends beyond the CT field of view, that part of the anatomy is truncated and not represented in the reconstructed CT image. Accordingly, there is no attenuation correction value for the corresponding region in the PET emission data; hence, introducing a bias on the PET attenuation corrected images of underestimation in the standardized uptake values in these regions. Truncation also produces streaking artifacts at the edge of the CT image, resulting in an overestimation of the attenuation coefficients used to correct the PET data, which in turn creates a rim of high activity at the truncation edge and potential misinterpretation of the PET examination [12].

In addition, lesions can be missed in parts of the liver that are affected by CT-based attenuation correction [13]. Attenuation correction can be applied erroneously on PET at the location of dense objects [14] or where the position of a transition from low to high photon-attenuating tissue does not correspond between PET and CT $[15,16]$. The diaphragmatic area is highly susceptible to such errors due to the combination of the sharp tissue/air transition and the likelihood of positional differences. The error may be apparent as a change in the hepatic contour on the attenuationcorrected PET images and a reduced sensitivity for lesions in the affected area. In addition, the PET signal is no longer quantitative in regions of attenuation-correction artifacts, which may compromise follow-up measurements [17].

FDG-PET/CT images are generally assessed qualitatively and quantitatively for pathologically increased radiotracer uptake. Standardized uptake value (SUV), a semiquantitative measurement, is widely used for this purpose. Partial volume effect can cause underestimation of the SUVmax of small tumors [18].

Lesion size also affects the sensitivity of PET imaging; those sized $<0.5-1.0 \mathrm{~cm}$ cannot be detected reliably [19, 20].

\section{Physiologic pitfalls}

The liver is the major producer of nondietary glucose (at a rate of $2.0 \mathrm{mg} / \mathrm{kg} / \mathrm{min}$ ), which helps maintain glucose homeostasis [1]. Normal liver parenchyma shows a certain degree of FDG uptake [21], having a mildly intense, uniformly mottled appearance. It can be difficult to distinguish between increased uptake related to a focal lesion and background physiological activity.

FDG uptake in tumors and tumor-like lesions is affected by blood glucose; i.e., SUV is inversely related to blood glucose, and normalization to blood glucose is recommended [22]. Body mass index affects 18 F-FDG uptake to a certain degree [23-25]. Factors such as age, sex, and diabetes status can also have a small influence on FDG uptake $[23,24]$ that can generally be ignored.

Finally, physiologic colonic activity abutting the margin of the liver should be taken into consideration [26].

\section{Malignant hepatic tumors}

The majority of primary and secondary malignant tumors show high FDG uptake. However, several tumors can potentially show false-negative uptake; even between tumors that are identical histologically, the degree of FDG uptake can differ according to tumor differentiation or proliferation rate [27-32]. In certain tumors, the degree of FDG uptake can be used to predict prognosis or therapeutic response [28, 29, 33-37].

\section{Hepatocellular carcinoma}

Hepatocellular carcinoma (HCC) is the most common primary malignancy of the liver, and occurs most commonly in chronic hepatitis and cirrhosis.

FDG accumulation is significantly higher in poorly differentiated HCCs than in well- and moderately differentiated HCCs [27] (Fig. 2), and is correlated with the expression of GLUT-1/-2 and HK-II, and G6Pase activity. Well- to moderately differentiated HCCs show low expression of GLUT1/-2 and high G6Pase activity; poor- and undifferentiated HCCs show high expression of GLUT-1/-2 and low G6Pase activity [38]. Therefore, the overall sensitivity of FDG-PET and FDG-PET/CT in detecting HCC has been relatively limited, with a reported range of 50-65\% [27, 28], because of poor detection of well- and moderately differentiated HCCs. In other words, FDG uptake can serve as a predictor of the grade of HCC differentiation.

The level of glycometabolic activity also has prognostic importance. High FDG uptake has been reported as an independent predictor of recurrence and poor survival after liver transplantation [33], and of poor response to radiation [34] and transarterial chemoembolization [35]. Although extrahepatic disease is relatively rare in HCC, especially in welldifferentiated tumors, its detection can significantly change patient management. FDG-PET/CT has been found to have a high sensitivity for the detection of lymph node and bone 


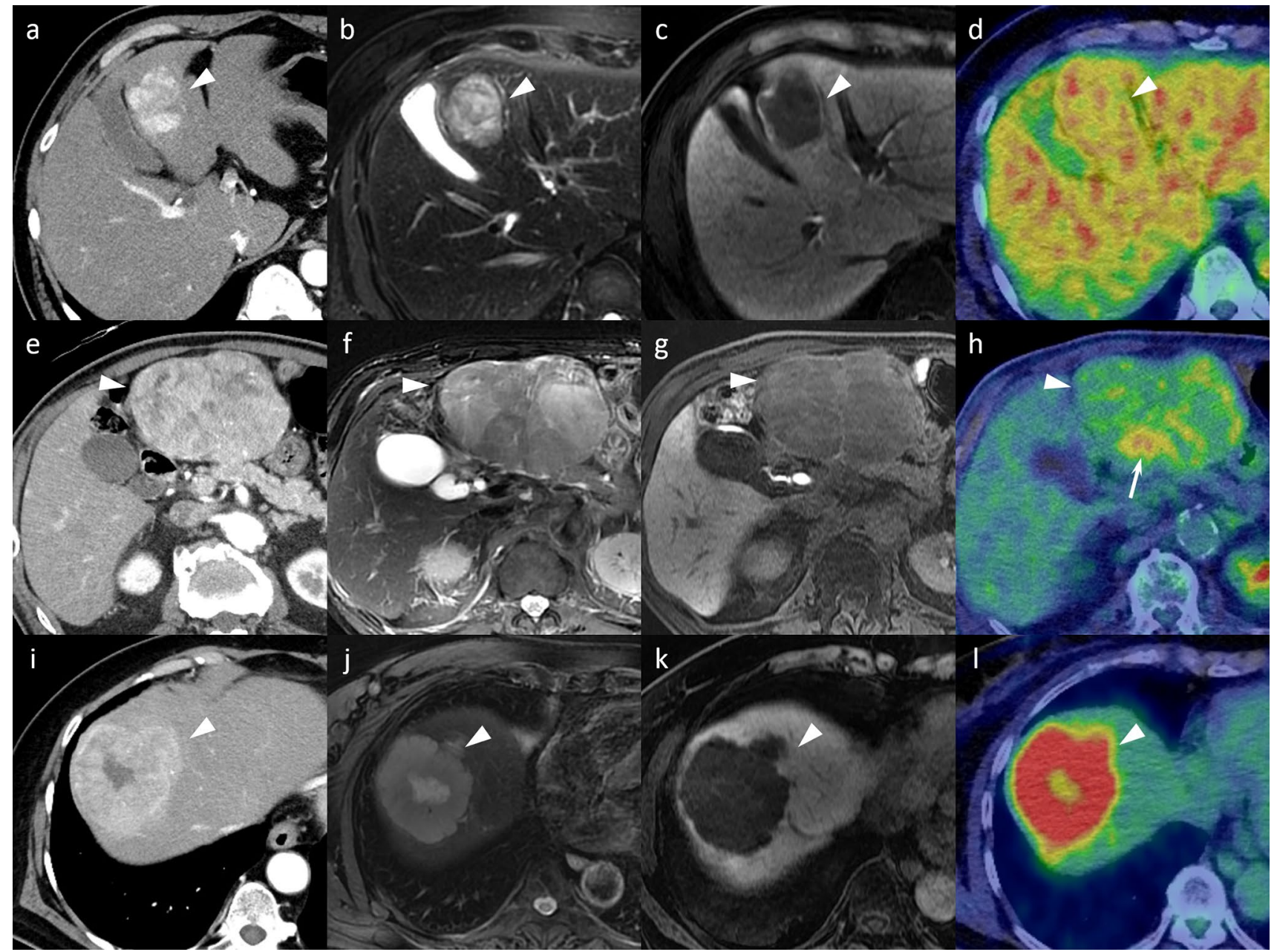

Fig. 2 FDG uptake in hepatocellular carcinoma (HCC) according to grade of differentiation. Well- to moderately differentiated HCC in a 63-year-old man with hepatitis B-virus-related cirrhosis. a Arterial phase CT reveals a hypervascular HCC (arrowhead). b Fat-suppressed T2-weighted MRI shows an inhomogeneous hyperintensity (arrowhead). c The tumor is apparent as an area of hypointensity (arrowhead) on hepatobiliary phase MRI. d On PET/CT, FDG uptake in the tumor (arrowhead) is similar to that of background liver. Moderately differentiated HCC of unknown etiology in an 81-year-old man with cirrhosis. e A hypervascular tumor (arrowhead) is evident on arterial phase CT. f The tumor shows inhomogeneous hyperinten-

metastases, and unsuspected extrahepatic metastases [39]. Thus, FDG-PET is a useful tool in predicting prognosis in HCC patients [36].

\section{Intrahepatic cholangiocarcinoma}

Intrahepatic cholangiocarcinoma (ICC) is the second most common primary liver neoplasm after HCC, and arises from the bile duct epithelium. ICC can be further subclassified into peripheral type (arising from small bile ducts) and perihilar type (arising from large bile ducts) based on the sity on fat-suppressed T2-weighted MRI (arrowhead). $\mathbf{g}$ The tumor is hypointense in the hepatobiliary phase (arrowhead). h PET/CT, FDG uptake is low (SUVmax 3.1) in a small portion (arrow) and slightly higher than background liver in most of the mass (arrowhead). Poorly differentiated HCC in a 75-year-old man with no history of chronic liver disease or cirrhosis. i Arterial phase CT reveals a large hypervascular HCC (arrowhead). j The tumor shows clear hyperintensity (arrowhead) on fat-suppressed T2-weighted MRI. k The tumor shows hypointensity (arrowhead) in the hepatobiliary phase. I PET/ CT reveals remarkably high FDG uptake (SUVmax 9.8) in the tumor (arrowhead) compared with background liver

location of the lesion; and into mass-forming and infiltrating types, based on the morphology. Therefore, the sensitivity of FDG-PET/CT in diagnosing ICC depends on both the location of the lesion and the morphological characteristics. More than $90 \%$ of ICCs show elevated FDG uptake that is particularly prominent in nodular or mass-forming cholangiocarcinoma located in the periphery [40] (Fig. 3a-d), and mass-forming ICC tends to show ring-like uptake, reflecting corresponding to peripheral rim enhancement on CT and/ or MRI [41]. The intense FDG uptake is due to increased expression of GLUT-1 and HK-II [38, 42]. Notably, the expression of GLUT-1 has a significant association with 


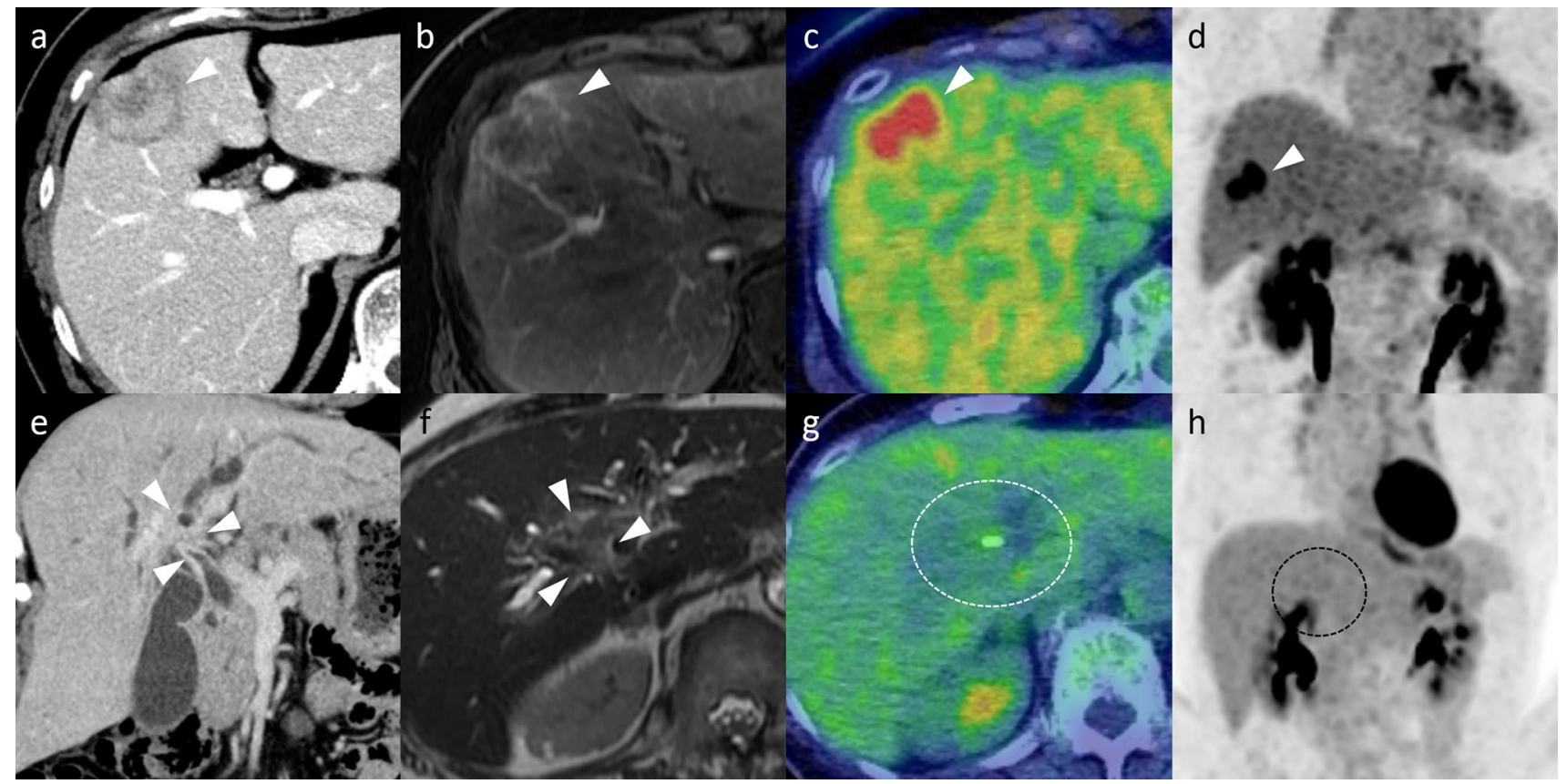

Fig. 3 Intrahepatic cholangiocarcinoma (ICC). Mass-forming type located in the peripheral area in a 75-year-old man (a-d) and infiltrating type located in the perihilar region in a 73-year-old woman (e-h). a Portal phase CT shows a moderately enhancing mass (arrowhead) with a target-like appearance. Capsular retraction is apparent. b On fat-suppressed T2-weighted imaging, the mass is hypointense with a peripheral hyperintense rim (arrowhead). c PET/CT reveals remarkably high FDG uptake (SUVmax 11.1) of the mass (arrowhead) compared with background liver. d Coronal maximum inten-

FDG uptake, especially in moderately and poorly differentiated ICC [42]. In contrast, perihilar and infiltrating type, which accounts for about $10 \%$ of all ICCs, has been reported to show lower uptake of FDG than peripheral and infiltrating type (Fig. 3e-h), and demonstrated increased FDG uptake of a focal nodular or linear branching appearance [41]. The finding may be associated with the smaller size and/or higher mucin content of hilar tumors compared with peripheral ones [40]. Therefore, FDG-PET/CT is accurate in predicting the presence of nodular type ICC, but less effective for infiltrating type.

Although FDG-PET/CT imaging has not been shown to be highly beneficial in terms of detectability of the primary lesion, it is beneficial in detecting unsuspected or unknown metastases. Thus, FDG-PET/CT staging has an important impact on the selection of therapy in $17-30 \%$ of patients $[43,44]$.

Cholangiolocellular subtype (also called cholangiolocellular carcinoma or bile ductular carcinoma), which had been classified as a type of combined hepatocellular cholangiocarcinoma (cHCC-CC) with stem cell features, is now classified as small duct type of intrahepatic cholangiocarcinoma according to World Health Organization classification sity projection (MIP) PET image shows high FDG uptake in the mass (arrowhead). The diagnosis of mass-forming ICC was confirmed by surgical resection. e Equilibrium phase CT shows an ill-defined perihilar mass and thickened bile duct wall (arrowheads). Dilatation of the peripheral bile duct is also apparent. $\mathbf{f}$ The ill-defined mass shows hypointensity on T2-weighted MRI (arrowheads). g No FDG uptake is detected in the mass on PET/CT (dotted circle). $\mathbf{h}$ No FDG uptake is detected in the mass on coronal MIP PET (dotted circle). The diagnosis of perihilar ICC was confirmed by biopsy and cytology

criteria (2019) [45]. Histologically, cholangiolocellular subtype is described as a well-differentiated adenocarcinoma that forms an anastomosing pattern of antler-like glands in edematous fine fibrous stroma and resembling a ductular reaction, that is seen in several acute and chronic liver diseases, including massive hepatic necrosis. Because of its extreme rarity, few studies have assessed the diagnostic performance of FDG-PET/CT for this subtype. It is considered that the biological nature of cholangiolocellular subtype has potential for false-negative accumulation of FDG [46] (Fig. 4), although further analysis is required for confirmation.

\section{Combined hepatocellular cholangiocarcinoma}

Combined hepatocellular cholangiocarcinoma (cHCC-CC) is a primary liver carcinoma defined by the unequivocal presence of both hepatocytic and cholangiocytic differentiation within the same tumor [45]. It is rare and has a poor prognosis, with a reported incidence ranging from 1.0 to $4.7 \%$ of all primary hepatic tumors. Because of its rarity, few 


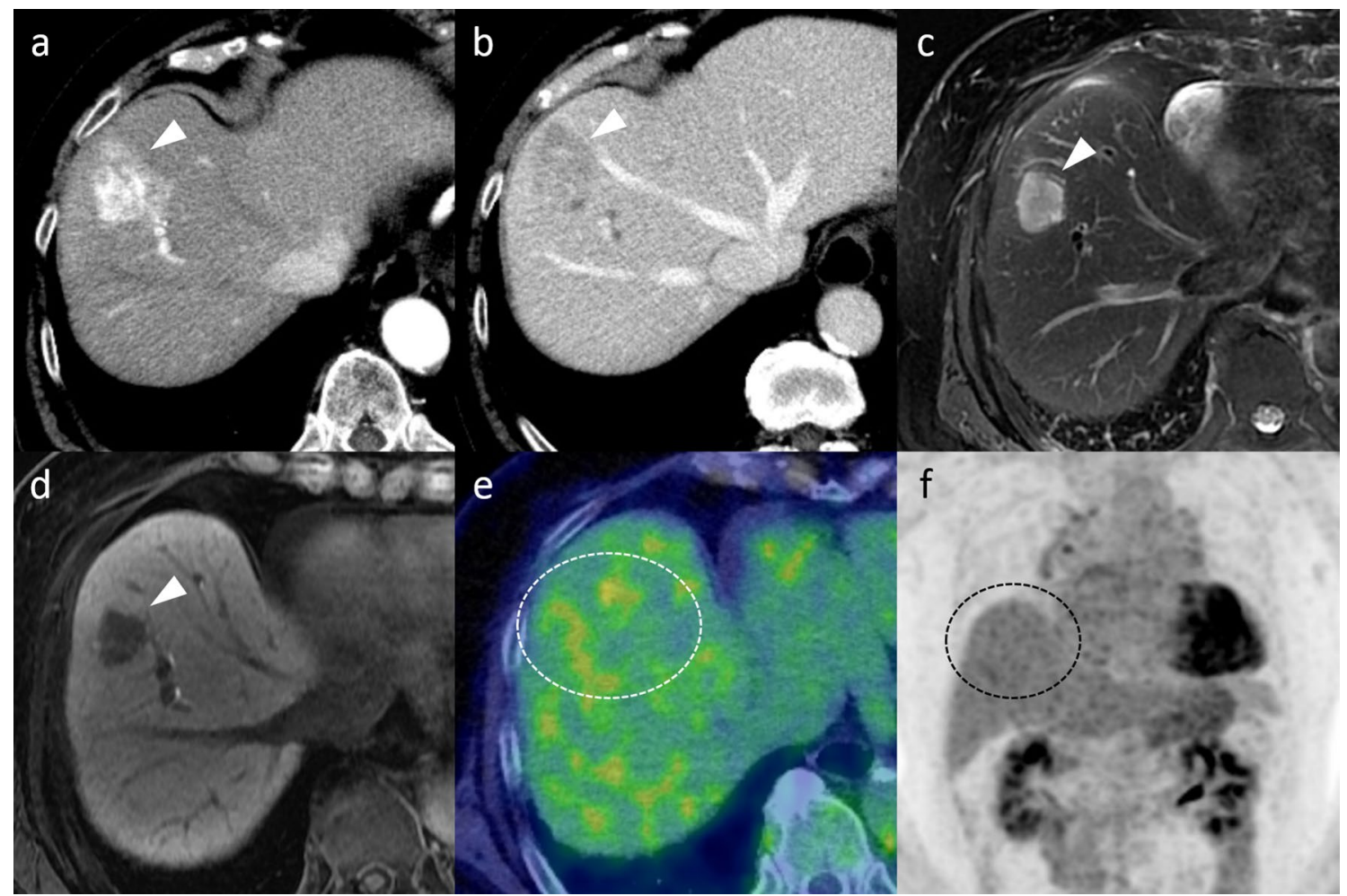

Fig. 4 Cholangiolocellular carcinoma in a 69-year-old man. a Arterial phase CT reveals an ill-defined and hypervascular hepatic mass (arrowhead). b On equilibrium phase CT, the mass is hypodense compared with background liver, and penetrating vessels are observed (arrowhead). c The mass shows hyperintensity on fat-suppressed T2-weighted MRI (arrowhead). d In the hepatobiliary phase,

studies have evaluated the accumulation of FDG and none to date has assessed the diagnostic performance of FDG-PET/ $\mathrm{CT}$ in cHCC-CC, although one has reported pretreatment tumor-to-normal liver SUV ratio as an independent predictor of survival in these patients [47]. As far as we know, the degree of FDG uptake depends on the respective histology. In other words, areas of hepatocytic component show various degrees of FDG uptake depending on differentiation, and areas of cholangiocytic component show high FDG uptake (Fig. 5).

\section{Hepatic angiosarcoma}

Primary hepatic angiosarcoma is a rare tumor originating from hepatic endothelial cells, and is characterized by its highly malignant nature and rapid progression. These tumors account for $\sim 1 \%$ of all primary hepatic carcinomas, but are the most common mesenchymal malignancy of the liver; metastatic hepatic angiosarcomas occur more frequently. Hepatic angiosarcomas are classified based on morphology as single mass, mixed massive mass, multiple nodules, and the mass shows hypointensity (arrowhead). e No FDG uptake is detected in the mass on PET/CT (dotted circle). f No FDG uptake is detected in the region of the mass on coronal MIP PET (dotted circle). The diagnosis of cholangiolocellular carcinoma was confirmed by percutaneous liver biopsy

diffuse nodule types, and it is common for more than one type to coexist. Most types of hepatic angiosarcoma share characteristic CT and MRI features of indistinct border, heterogeneous density, and frequent intratumoral hemorrhage; and typical centripetal heterogeneous enhancement on enhanced imaging. In addition, highly malignant angiosarcoma shows no enhancement due to intratumoral hemorrhage, cystic degeneration, and necrosis [48].

Single mass and mixed massive mass types of hepatic angiosarcoma generally show high FDG uptake [49, 50], which is considered to be related to overexpression of GLUT-1 [48]; and diffuse nodule type sometimes shows a false-negative accumulation of FDG due to lower cellular density, because of intratumoral hemorrhage and necrosis [51].

\section{Malignant lymphoma}

Malignant lymphoma, divided broadly into Hodgkin's and non-Hodgkin's lymphoma, encompasses a wide range of malignant diseases with differing histologic characteristics, 


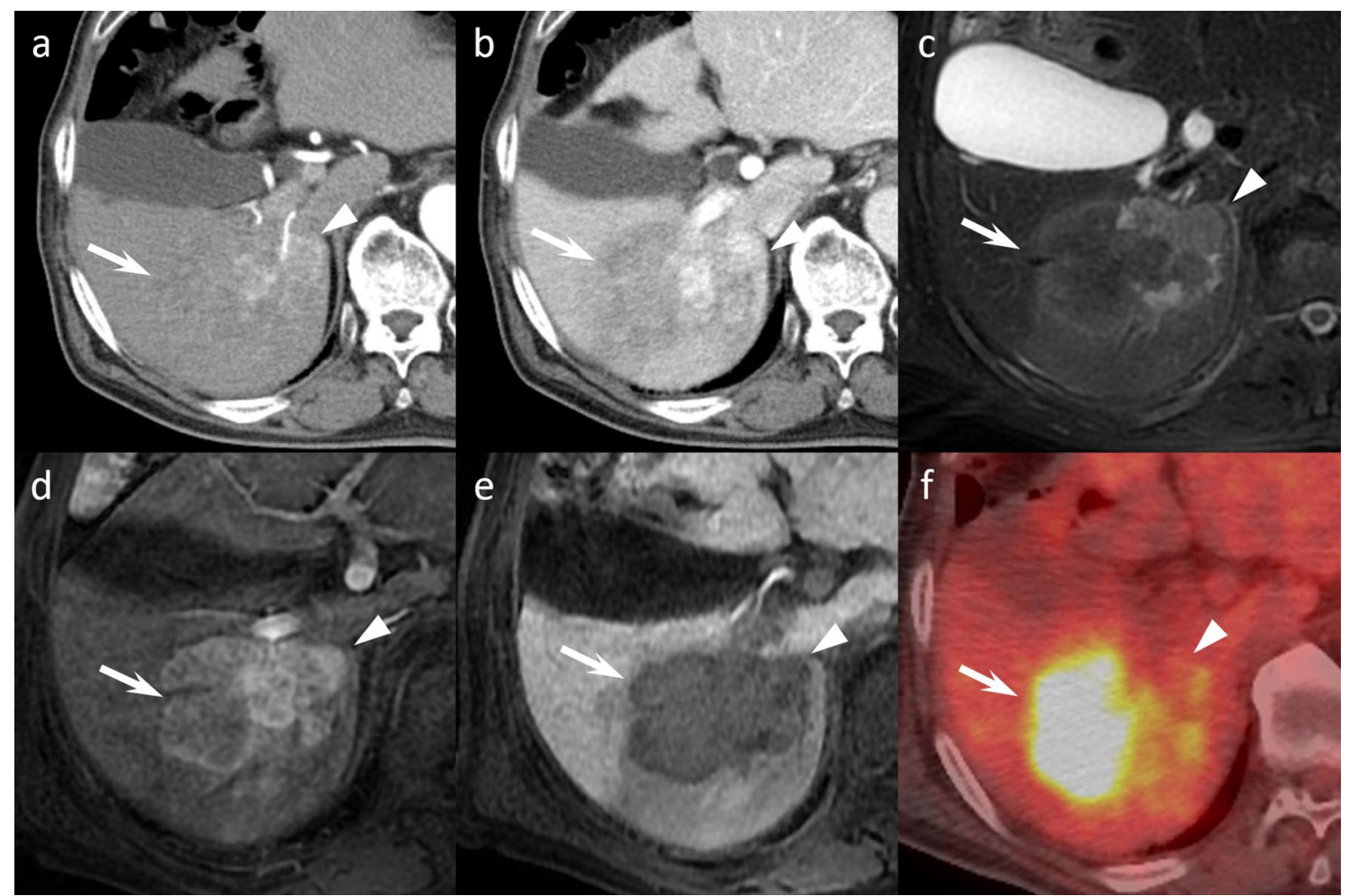

Fig. 5 Combined hepatocellular cholangiocarcinoma in an 81-yearold man with a history of sigmoid colon cancer. a Arterial phase CT shows a large mass comprising hypervascular (arrowhead) and hypovascular (arrow) components. The border between the two components is indistinct. b Portal phase CT reveals areas of persistent enhancement (arrowhead) and hypovascularity (arrow). c On fat-suppressed T2-weighted MRI, the area of persistent enhancement shows hyperintensity (arrowhead), whereas the hypovascular area shows hypointensity (arrow). d The areas of persistent enhancement (arrowhead) and hypovascularity (arrow) are apparent on portal phase MRI.

behavior patterns, imaging appearances, and treatment options. Non-Hodgkin's and Hodgkin's lymphomas rank as the 13th and 28th most frequent cancers worldwide, respectively; in 2018, an estimated 509,590 and 79,990 new cases were diagnosed and there were 248,724 and 26,167 deaths due to the respective lymphomas. From the clinical viewpoint, clinicopathologic grading of cases into indolent, aggressive, or highly aggressive is a useful system that broadly correlates with the management approach and FDG avidity [29] (Fig. 6). The most common of the aggressive and highly aggressive non-Hodgkin's lymphomas is diffuse large B-cell lymphoma; indolent (low grade) lymphomas include mucosa-associated lymphoid tissue, chronic lymphocytic leukemia/small lymphocytic lymphoma, marginal zone lymphoma, and peripheral T-cell lymphoma [30].

Secondary hepatic involvement by lymphoma is not uncommon, especially in patients with non-Hodgkin's lymphoma; whereas primary hepatic lymphoma is extremely rare, accounting for approximately $0.016 \%$ of e In the hepatobiliary phase, the whole mass displays hypointensity. $\mathrm{f}$ PET/CT reveals no uptake in the area of persistent enhancement (arrowhead), and high FDG uptake in the hypovascular area (SUVmax 8.7) (arrow). The diagnosis of combined hepatocellular cholangiocarcinoma was confirmed by surgical resection. Histologically, the area of persistent enhancement comprised mainly cells with hepatocytic differentiation and cholangiolocellular-type cells, whereas cells in the hypovascular area showed characteristics of cholangiocytic differentiation

all non-Hodgkin's lymphoma [52], and the most common subtype is diffuse large B-cell lymphoma [53]. Primary hepatic lymphoma can be classified morphologically into two distinct patterns, nodular (more frequent) and diffuse liver infiltration (less frequent), and generally presents as a solitary nodular lesion [53]. In a previously undiagnosed homogeneous solitary hepatic mass, extremely high FDG uptake in the lesion is suggestive of lymphoma, especially diffuse large B-cell lymphoma (Fig. 6a-d). In addition, in the case of diffuse infiltration of primary hepatic lymphoma, the CT and MRI findings are similar to those of acute hepatitis [54], and high FDG uptake in the whole liver indicates lymphoma; in this situation, the FDG-PET findings are useful in deciding whether percutaneous liver biopsy should be performed and in indicating an appropriate biopsy location for the higher activity of the lesions (Fig. 7).

FDG-PET/CT imaging is used in staging, assessment of therapeutic response, and early detection of disease recurrence in malignant lymphoma. The degree of FDG uptake correlates 


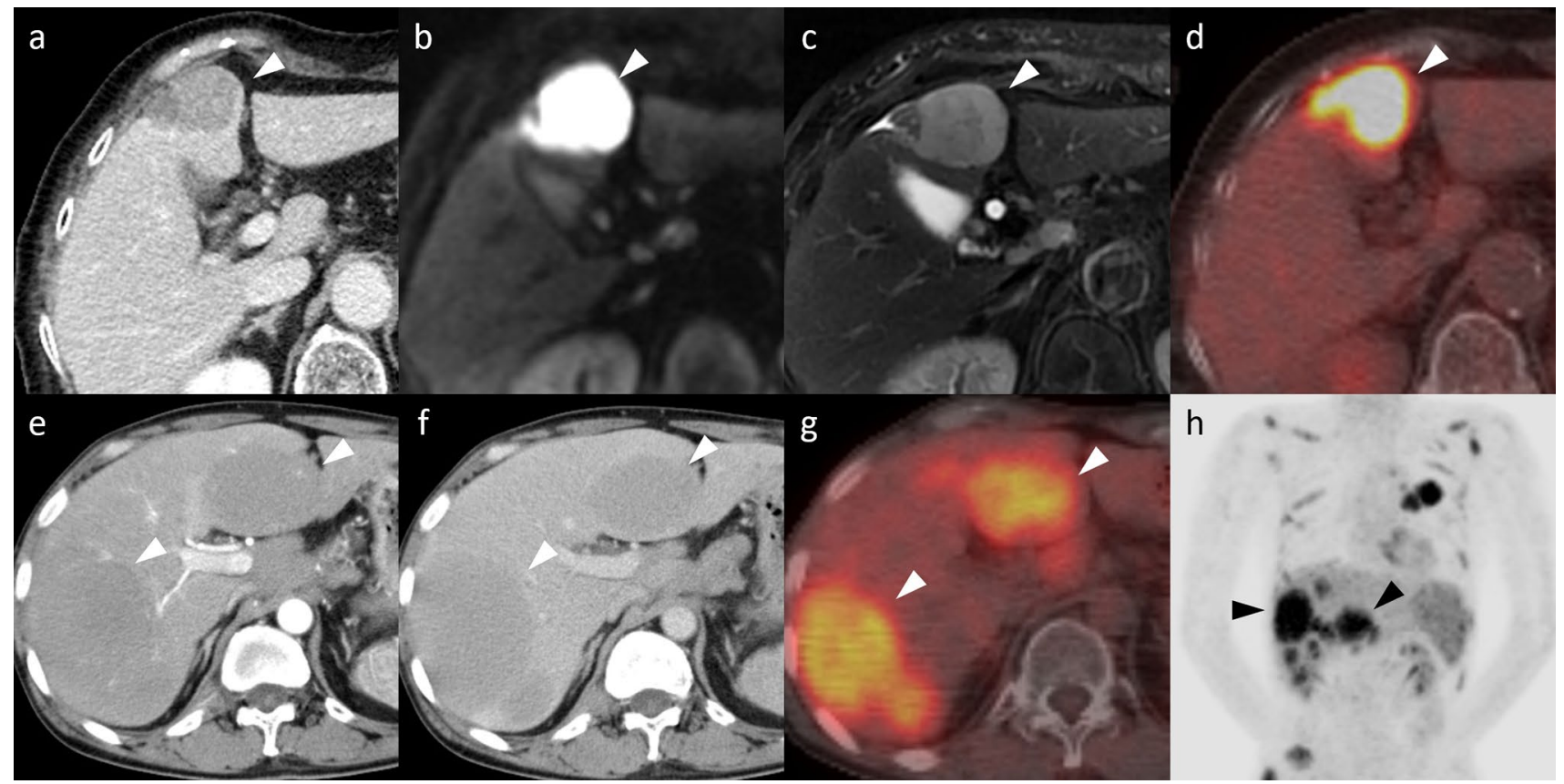

Fig. 6 Primary hepatic diffuse large B-cell lymphoma (aggressive lymphoma) in a 50-year-old man, and hepatic involvement of mucosa-associated lymphoid tissue lymphoma (indolent lymphoma) in a 61-year-old man. a Equilibrium phase CT reveals a solitary hypovascular hepatic mass (arrowhead). b The mass is hyperintense on diffusion-weighted MRI (value of b 800) (arrowhead). $\mathbf{c}$ The mass is also hyperintense on fat-suppressed T2-weighted MRI (arrowhead). d PET/CT reveals extremely high FDG uptake (SUVmax 21.4) in the mass (arrowhead). There was no other area of abnormal uptake in the whole body. The diagnosis of diffuse large B-cell lymphoma was con- firmed by percutaneous liver biopsy. e Arterial phase CT reveals multiple homogeneous and hypovascular hepatic masses (arrowheads). $f$ The masses also appear homogeneous and hypovascular on equilibrium phase CT (arrowheads). g PET/CT shows relatively high FDG uptake (SUVmax 7.4) in the masses (arrowheads). h FDG uptake in multiple hepatic masses (arrowheads), multiple lymph nodes, and bone marrow is apparent on coronal MIP PET. The diagnosis of mucosa-associated lymphoid tissue lymphoma was confirmed by lymph node biopsy with the level of aggressiveness. In general, high FDG uptake is seen in aggressive lymphoma, whereas indolent lymphoma is associated with lower FDG uptake [55]. Furthermore, there is strong evidence that increased FDG avidity correlates with poor prognosis and poor response to treatment $[56,57]$. The presence of high FDG uptake in a patient with known lowgrade lymphoma indicates high-grade transformation (termed Richter's transformation) [58].

Regarding the molecular background, GLUT-1 and GLUT-3 contribute to FDG uptake in diffuse large B-cell lymphoma and in extranodal natural Killer/T-Cell lymphoma. SUV max and GLUT-3 may have a relationship with the prognosis of diffuse large B-cell lymphoma. In patients with Hodgkin's lymphoma, high expression of GLUT-1 is related to FDG uptake [59-61].

\section{Primary and metastatic neuroendocrine tumors}

Neuroendocrine neoplasms are rare; they have great heterogeneity and can originate from peptidergic neurons and neuroendocrine cells throughout the body. The most common sites of these tumors include the gastrointestinal tract, lung, adrenal gland, and thyroid gland. The most common sites of metastasis are the lymph nodes, liver, spleen, and bone.

Primary hepatic neuroendocrine tumors are extremely rare, and liver metastasis commonly develops in gastroenteropancreatic neuroendocrine tumors. According to the grade of differentiation based on proliferation index 


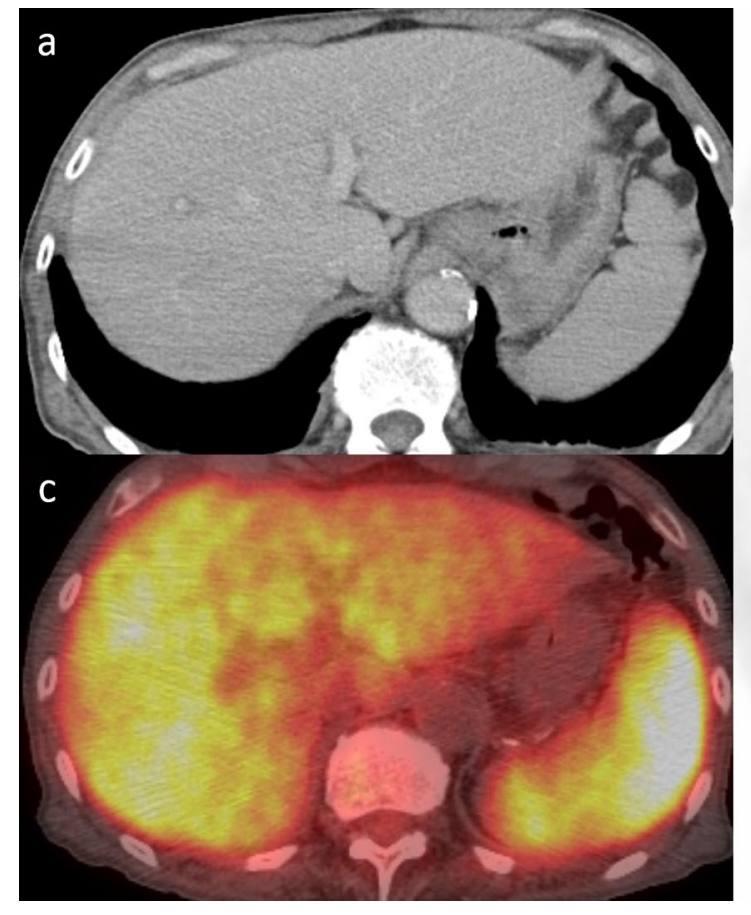

Fig. 7 Diffuse type of hepatic involvement of diffuse large B-cell lymphoma in an 84-year-old man. a Equilibrium phase CT reveals no obvious abnormalities such as hepatomegaly or areas of abnormal enhancement. b Coronal MIP PET shows diffuse uptake of FDG in the liver, spleen, and bone marrow. An adequate sample of bone marrow was obtained by biopsy prior to the PET examination. c PET/

(Ki-67), neuroendocrine tumors are classified as follows: well- or moderately differentiated neoplasms are defined as G1 (Ki-67 $\leq 2 \%$ ), G2 (Ki-67 3\%-20\%), or G3 (Ki-67 > 20\%); and poorly differentiated neoplasms are defined as neuroendocrine carcinoma (Ki-67 > 20\%) [62].

FDG uptake is correlated with neuroendocrine tumor aggressiveness, and GLUT-1 expression has a significant relation to FDG accumulation [63]. The sensitivity of PET/CT can differ according to the grade of differentiation, which is relatively low in G1 or G2 neoplasms and higher in poorly differentiated neuroendocrine carcinomas. Thus, the overall sensitivity of PET/CT in detecting whole-body primary and metastatic neuroendocrine tumors that include the liver has been shown to be relatively limited, with a reported range of only 36-52\% [31, 32] (Fig. 8). However, PET/CT can provide prognostic information [31]. Previous studies have reported that $\mathrm{PET} / \mathrm{CT}$ is a predictor of progression-free survival [37], and multivariate analysis revealed that $\mathrm{SUVmax}>3$ is an independent predictor of progression-free survival [32].

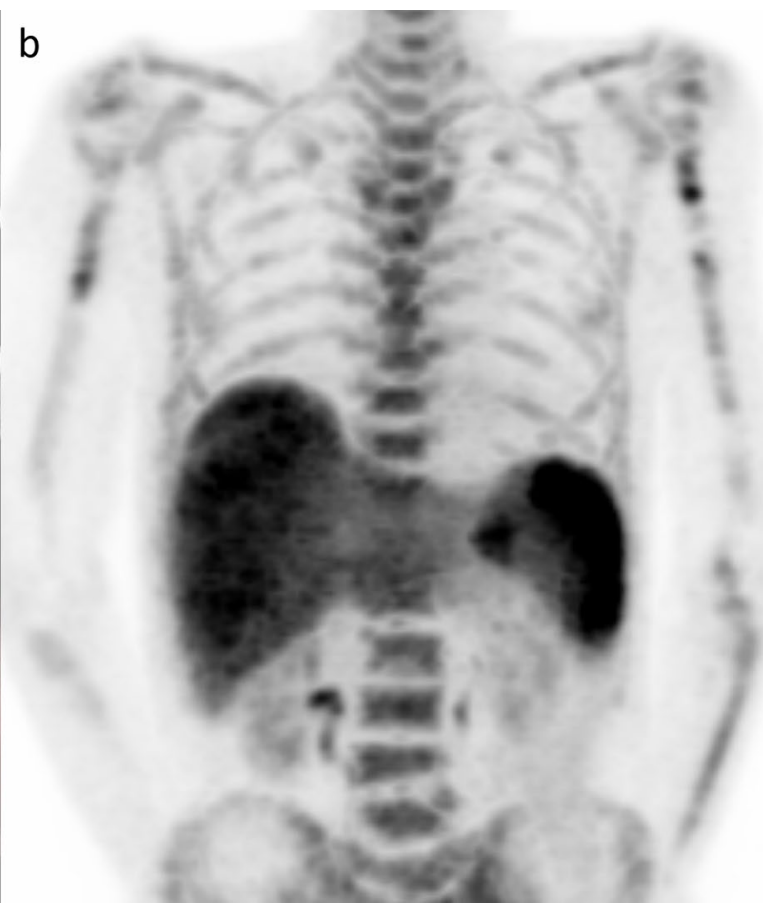

CT reveals diffuse uptake of FDG in the liver and spleen. In addition, the uptake of the liver is heterogeneous, and the uptake in the right lobe is higher than that in left lobe. PET images clarify the hepatic involvement and can guide the appropriate biopsy location. The diagnosis of diffuse large B-cell lymphoma was confirmed by percutaneous liver biopsy from the right lobe

\section{Hepatic metastasis}

The liver is involved more often with metastatic disease than primary liver tumors, being a common site of metastasis of tumors arising from such as the gastrointestinal tract, lung, breast, pancreas, as well as sarcoma. The accurate detection and characterization of hepatic metastases are crucial because they decide the treatment strategy.

Although several studies have reported the superiority of PET/CT over CT in the detection of untreated or unexpected hepatic metastases from various primary cancers [64, 65], gadolinium ethoxybenzyl diethylenetriamine pentaacetic acid (Gd-EOB-DTPA)-enhanced MRI has superiority over both dynamic contrast-enhanced CT and combined FDG-PET/CT [66, 67], especially in detecting small lesions. However, FDG-PET/CT has the advantage of simultaneous detection of extra-hepatic lesions, and is widely used in the assessment of various malignant tumors. 


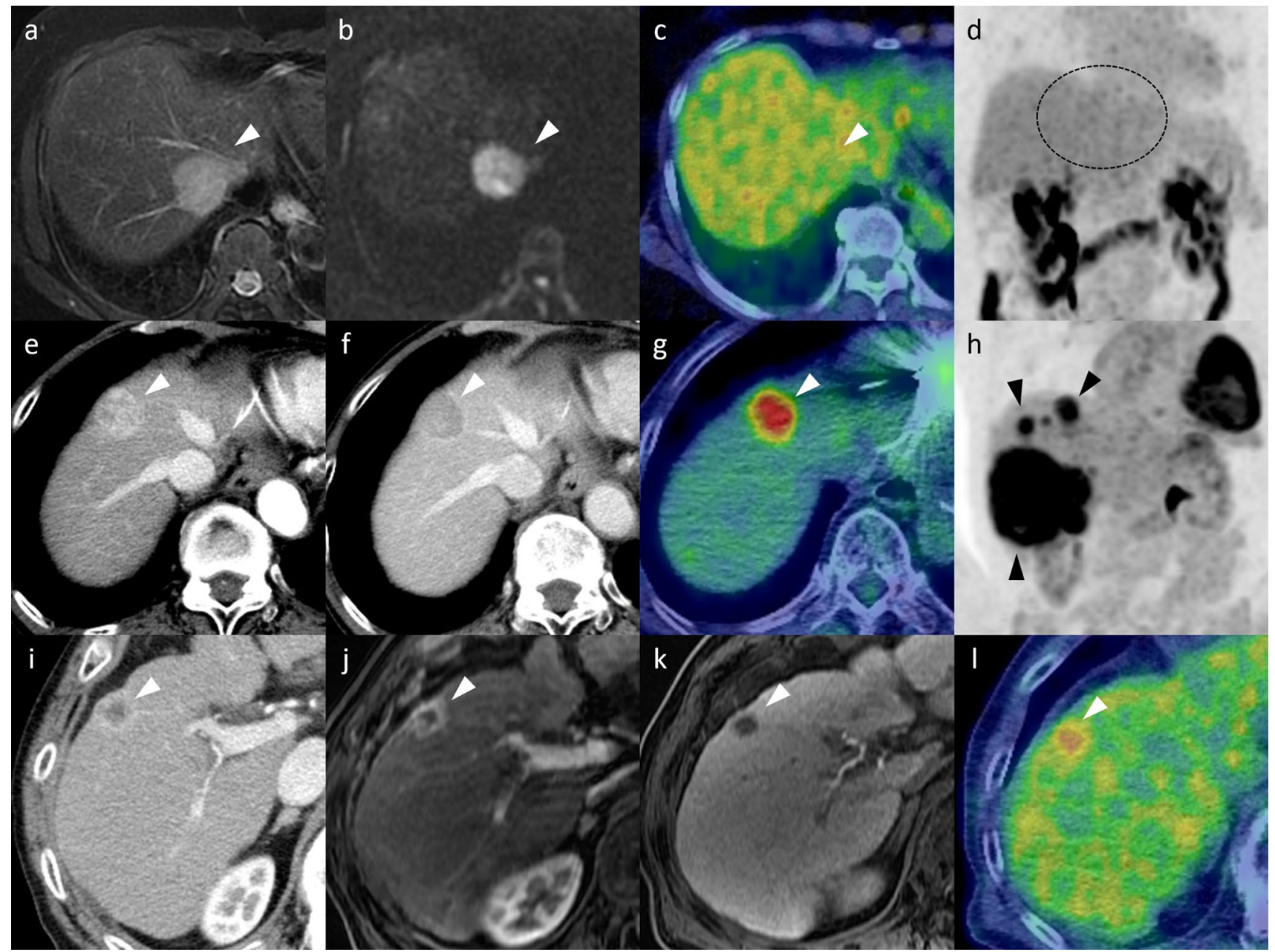

Fig. 8 FDG uptake in primary neuroendocrine tumor according to grade of differentiation. Primary neuroendocrine tumor (G2, Ki-67 $5 \%$ ) in a 73-year-old man. a Fat-suppressed T2-weighted MRI reveals a hyperintense mass (arrowhead). b The mass is also hyperintense on diffusion-weighted MRI (value of $b$ 800) (arrowhead). c PET/CT shows no FDG uptake in the mass (arrowhead). d Coronal MIP PET also shows no FDG uptake in the mass (dotted circle). Primary neuroendocrine tumors (G2, Ki-67 10\%) in an 80-year-old man. e Arterial phase CT reveals a hypervascular mass (arrowhead). f The mass is hypodense compared with background liver on portal phase CT (arrowhead). g On PET/CT, the mass (arrowhead) shows high FDG uptake (SUVmax 7.6). h Coronal MIP PET reveals high

Metastases generally present with a similar biological nature to that of the primary tumor, and most hepatic metastasis show high uptake of FDG [66, 67], which is related to the overexpression of GLUT-1 in primaries in such as the lung, pancreas, colorectum, breast, and biliary tract [68]. The degree of FDG uptake depends fundamentally on the differentiation or histopathologic characteristics of the tumor $[69,70]$. It is important to note that some of these tumors may show low uptake or false-negative uptake of FDG.
FDG uptake in multiple hepatic masses (arrowheads). Primary poorly differentiated neuroendocrine tumor (neuroendocrine carcinoma, Ki-67 index 50\%) in a 62-year-old man. i Portal phase CT reveals a mass with peripheral dominant enhancement. The central hypovascular area indicates degeneration of the tumor (arrowhead). j Arterial phase MRI also shows peripheral dominant enhancement of the mass (arrowhead). $\mathbf{k}$ The mass shows hypointensity in the hepatobiliary phase (arrowhead). l On PET/CT, the mass (arrowhead) shows moderate FDG uptake (SUVmax 4.1). Note that the FDG uptake is relatively low due to severe degeneration of the neuroendocrine carcinoma.

\section{Low-glucose-metabolizing tumors due to well-differentiated tumor}

Some tumors have low glucose metabolism, particularly those that are well differentiated, including pancreatic adenocarcinomas [71], prostate cancer [72], and lung adenocarcinoma [73]; bronchioloalveolar carcinoma of the lung [74], and neuroendocrine tumor (G1) [31, 37]. Hepatic metastases of these tumors can have relatively low FDG uptake as with the primary tumor, and their FDG uptake 
can be masked by the mottled appearance of background liver uptake.

\section{Low cellularity due to mucin, cystic component, degeneration, and intratumoral hemorrhage}

In cystic tumors, and in those with mucin, degeneration, or cystic change within the tumor, there are fewer viable cancer cells, which can yield low FDG uptake due to their low cellularity [74].

The tumor most likely to metastasize to the liver is mucinous carcinoma, commonly found in the gastrointestinal tract and accounting for approximately $17 \%$ of colonic tumors, and also found in the ovary. These tumors contain clear, gelatinous fluid (mucin) that can be intracellular or extracellular. FDG uptake directly correlates with the number of viable cancer cells; therefore, mucinous tumors with few viable cancer cells may not demonstrate increased FDG avidity. PET/CT is limited in the evaluation of mucinous tumors (Fig. 9), particularly in hypocellular lesions with abundant mucin, for which PET/CT has been reported to have sensitivity of 59\% [75].

Adenoid cystic carcinoma (ACC) is an uncommon form of adenocarcinoma that arises within secretory glands, most commonly the major and minor salivary glands of the head and neck, accounting for 5-10\% of all salivary gland tumors. ACC tends to invade the nerves and cause distant hematogenous metastasis, the preferential sites of which are the lung and bone, followed by the brain and liver. PET/CT has limited ability to detect metastases from adenoid cystic carcinoma for the same reasons as for mucinous carcinoma [76] (Fig. 10).

Degeneration or intratumoral hemorrhage in any tumor can yield low FDG uptake (Fig. 11).

\section{Renal cell carcinoma}

Renal cell carcinoma (RCC) is one of the most prevalent tumors worldwide. Clear cell RCC (ccRCC) is the most common histologic type. CT is considered the gold standard for characterization of renal tumors, although MRI can be used to characterize renal tumors that are indeterminate on US or CT. In contrast, FDG-PET/CT can detect fewer than half of all primary and metastatic RCCs [77]. SUVmax is significantly lower in low-grade ccRCC than in high-grade ccRCC [77]. FDG uptake in RCC is associated with the expression of GLUT-1 [78, 79], and there is a positive association between SUVmax and GLUT-1 expression in ccRCC [79]. Low G6Pase activity due to decreased expression of the G6Pase catalytic subunit would cause low uptake of FDG in RCC; however, the expression of G6PTs is reported to vary, and no general trend has been found between tumorous and non-tumorous tissues [80] (Fig. 1).

\section{Malignant melanoma}

Malignant melanoma, which develops from pigment-containing melanocytes, typically occurs in the skin, but may occur rarely in the mouth, intestines, or eye. It has a notoriously poor prognosis due to the high rates of nodal and distant metastases and recurrence. The lymph nodes are the most common site of metastasis $(73.6 \%)$, followed by the liver $(58.3 \%)$, brain $(54.6 \%)$, bone $(48.6 \%)$, and adrenal glands (46.8\%). In uveal melanoma, however, the liver is the most common (and often the only) site of systemic metastasis, due to the different biological behaviors between cutaneous and uveal melanoma.

PET/CT imaging is established as sensitive and accurate method for diagnosing primary and metastatic lesions of

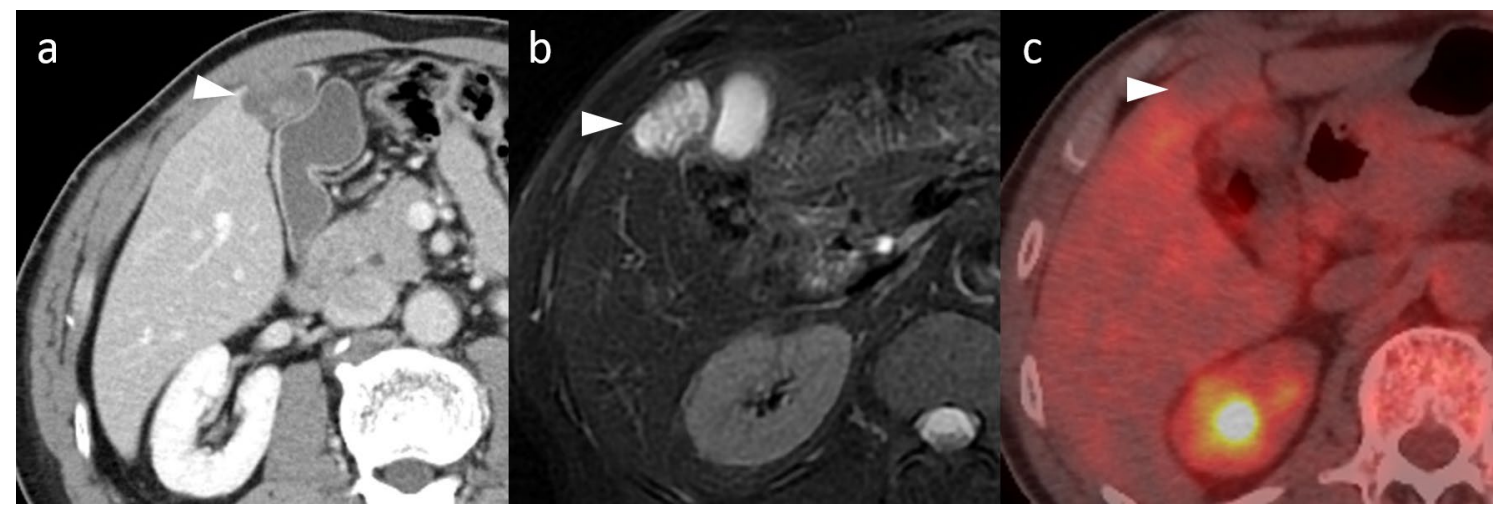

Fig. 9 Hepatic metastasis of advanced rectal mucinous adenocarcinoma in a 77-year-old man. a Equilibrium phase CT reveals a hypovascular hepatic mass (arrowhead). b On fat-suppressed T2-weighted MRI, the mucinous component of the mass shows obvious hyperin- tensity (arrowhead). c No uptake of FDG is seen on PET/CT (arrowhead). The diagnosis of hepatic metastasis of mucinous adenocarcinoma was confirmed by surgical resection. Abundant mucin within the tumor can yield low FDG uptake due to low cellularity 


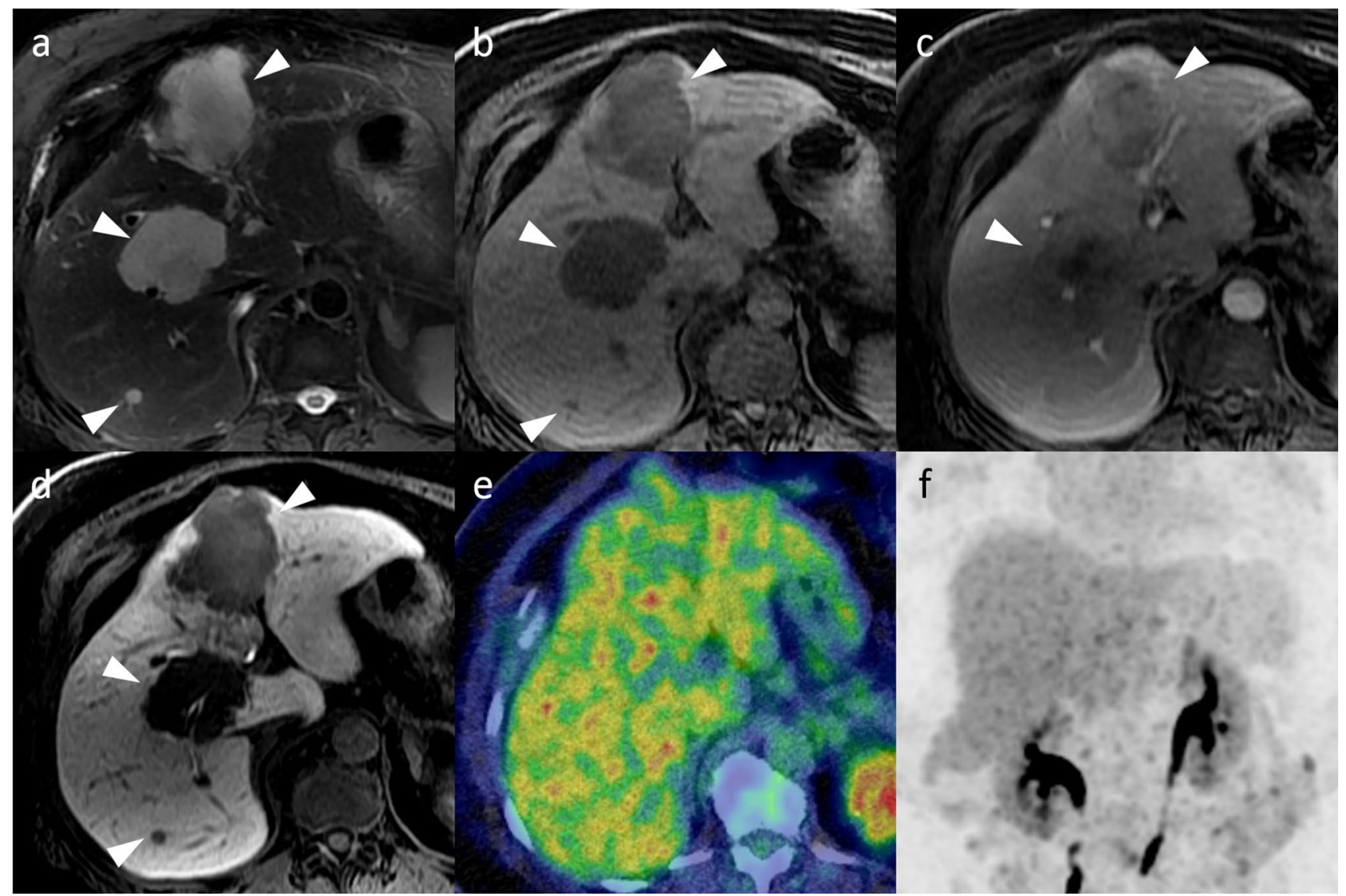

Fig. 10 Multiple metastases of submandibular adenoid cystic carcinoma in a 52-year-old woman. a Fat-suppressed T2-weighted MRI depicts multiple hyperintense hepatic masses (arrowheads). b Precontrast T1-weighted MRI of a dynamic contrast study reveals multiple hypointense hepatic masses (arrowheads). c The masses show

cutaneous melanoma [81], but does not appear to be sensitive enough to detect uveal melanoma [82,83].

The expression of GLUT-1 is related to the FDG uptake of melanoma; however, $60 \%$ of hepatic metastases of uveal melanoma show negative uptake of FDG, and there is no obvious difference in expression of GLUT-1 between hepatic metastases from cutaneous and uveal melanoma [84] (Fig. 12). The reason for this discrepancy is unclear, but the low FDG uptake may be related to an unknown mechanism of glycometabolic disorder.

\section{Benign hepatic tumors and tumor-like lesions}

The majority of benign tumors and non-tumorous lesions show no FDG uptake, or uptake similar to that of background liver [85]; however, benign tumors and inflammatory and infectious lesions can show false-positive uptake [86-89]. In the case of equivocal CT and MRI findings, low moderate enhancement in the portal phase of the dynamic contrast study (arrowheads). d The masses show hypointensity in the hepatobiliary phase (arrowheads). e PET/CT shows no FDG uptake in the masses. f Coronal MIP PET also shows no FDG uptake in the masses. The diagnosis of metastasis was confirmed by surgical resection

or no FDG uptake can assist in differentiating benign tumor from malignancy $[85,90]$.

\section{Hepatocellular adenoma}

Hepatocellular adenoma (HCA) is rare benign liver neoplasm that occurs predominantly in women of reproductive age. It is classified into the following molecular subgroups that are linked with certain risk factors, clinical behaviors, histological features, and imaging: hepatocyte nuclear factor $(\mathrm{HNF})-1 \alpha$-inactivated HCA, inflammatory HCA, CTNNB1-mutated HCA in exon 3, CTNNB1-mutated HCA in exon 7 or 8, sonic hedgehog HCA, and unclassified HCA. CTNNB1-mutated HCA in exon 3 and sonic hedgehog HCA have been linked with high risk of malignant transformation and bleeding, respectively [91]. Characteristic imaging findings, especially those of EOB-enhanced MRI, may clarify the subtype and enable distinction of HCA from other hypervascular hepatic tumors $[92,93]$. 


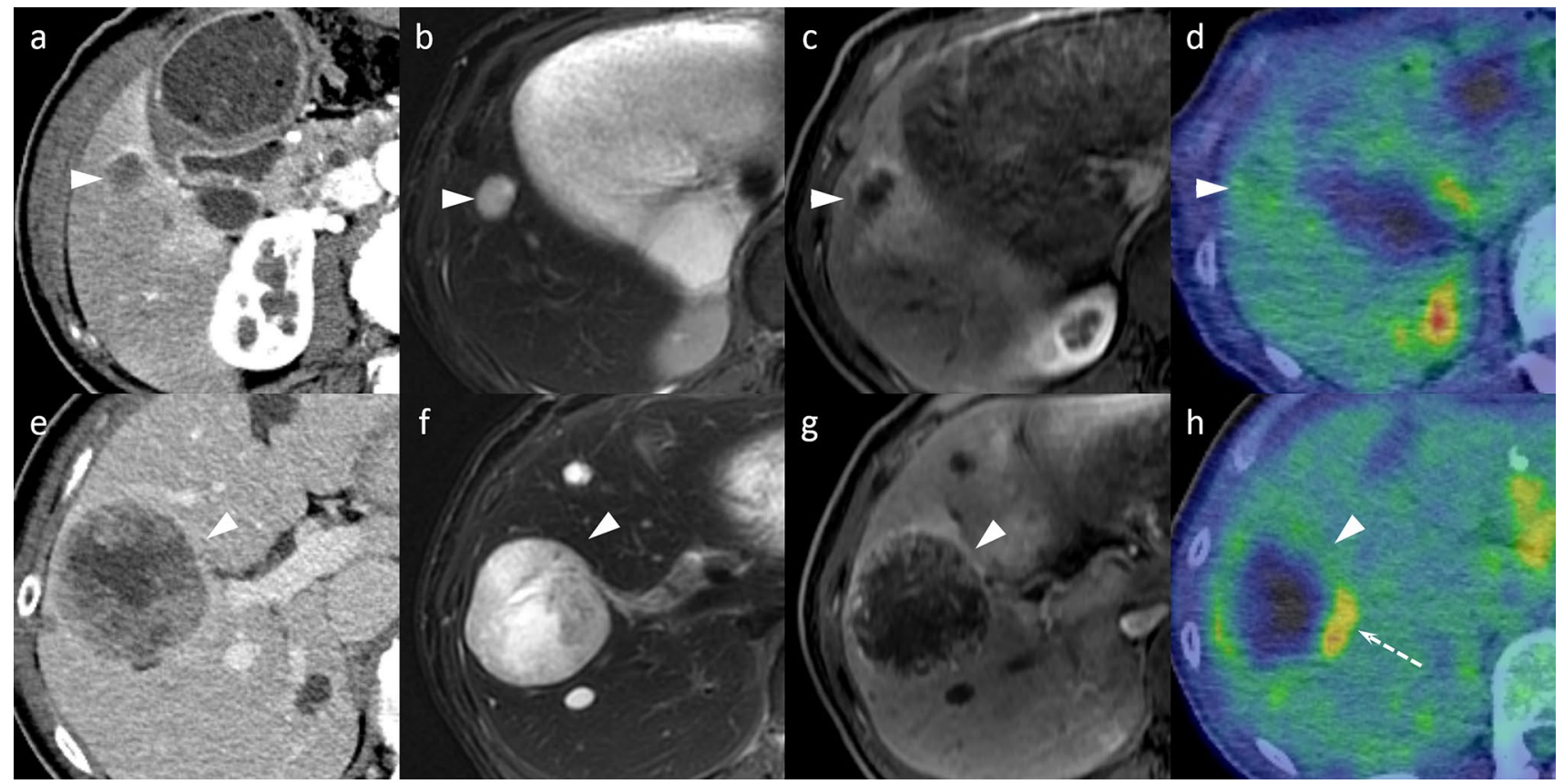

Fig. 11 Multiple metastases of gastric gastrointestinal stromal tumor (GIST) in a 55-year-old man. a Arterial phase CT reveals a hypovascular nodule (arrowhead). b The nodule shows obvious hyperintensity on fat-suppressed T2-weighted MRI (arrowhead). c The nodule can be seen on arterial phase MRI (arrowhead). d No FDG uptake is detected in the nodule on PET/CT (arrowhead). e Arterial phase CT reveals another hypovascular mass (arrowhead). f The mass

In general, HCA other than HNF-1 $\alpha$-inactivated type shows similar accumulation of FDG to that of background liver [85, 87, 94] because HCA is composed of cells that function similarly to normal hepatocytes: FDG is taken into the cytoplasm by GLUT-2 and is phosphorylated by HK-IV. FDG-6-phosphate is dephosphorylated by G6Pase as in normal hepatocytes. Dephosphorylated FDG is transported out of the cell by GLUT to maintain an equilibrium state.

However, it is noteworthy that HNF-1 $\alpha$-inactivated HCA shows high FDG uptake (Fig. 13) [88, 89]. In this subtype, GLUT-2, HK-IV, and G6Pase are expressed as in other subtypes of HCA and normal hepatic tissue. The inactivation of G6PT1, which is transcriptionally regulated by HNF1 $\alpha$ [95], prevents the uptake of phosphorylated FDG into the endoplasmic reticulum; accordingly, phosphorylated FDG is not dephosphorylated and is instead retained in HNF-1 $\alpha$-inactivated HCA cells, although G6Pase expression does not decrease [88] (Fig. 1).

To the best of our knowledge, no previous reports have described FDG uptake in the other HCA subtypes. FDGPET could possibly have clinical value in enabling the discrimination of HNF- $1 \alpha$-inactivated HCA from other HCA subtypes and other fat-containing hypervascular tumors. shows obvious hyperintensity on fat-suppressed T2-weighted MRI (arrowhead). g The mass appears hypovascular on arterial phase MRI (arrowhead). h No FDG uptake is observed in most of the mass (arrowhead), except in a small portion (SUVmax 4.6) (arrow). Percutaneous liver biopsy confirmed the diagnosis of severely degenerated metastasis of GIST. Degeneration of the tumor can yield low FDG uptake due to its low cellularity

\section{Hepatic cavernous hemangioma and sclerosed hemangioma}

Cavernous hemangioma is the most common benign hepatic tumor, with a reported incidence as high as $20 \%$. These tumors contain vascular channels of various sizes that are lined by a single layer of endothelial cells and supported by a collagenous wall. In most hemangiomas, the typical imaging findings (particularly those of MRI) are highly reliable for diagnosis [96] and remain stable on follow-up imaging [98].

Hemangiomas show similar uptake of FDG to background liver $[85,90,98]$. In patients with a history of malignant tumor, the finding of remarkable enlargement of a hemangioma showing poor uptake of FDG differentiates the lesion from metastatic disease $[85,90]$.

Slow blood flow within a hemangioma can cause degenerative change such as thrombus, necrosis, fibrosis, calcification, and scar formation, and hemangioma with these features is termed sclerosed hemangioma. These pathological changes can mask the typical imaging findings on CT and MRI; thus, sclerosed hemangioma can be misdiagnosed as malignant tumor such as ICC, hepatic metastases, 


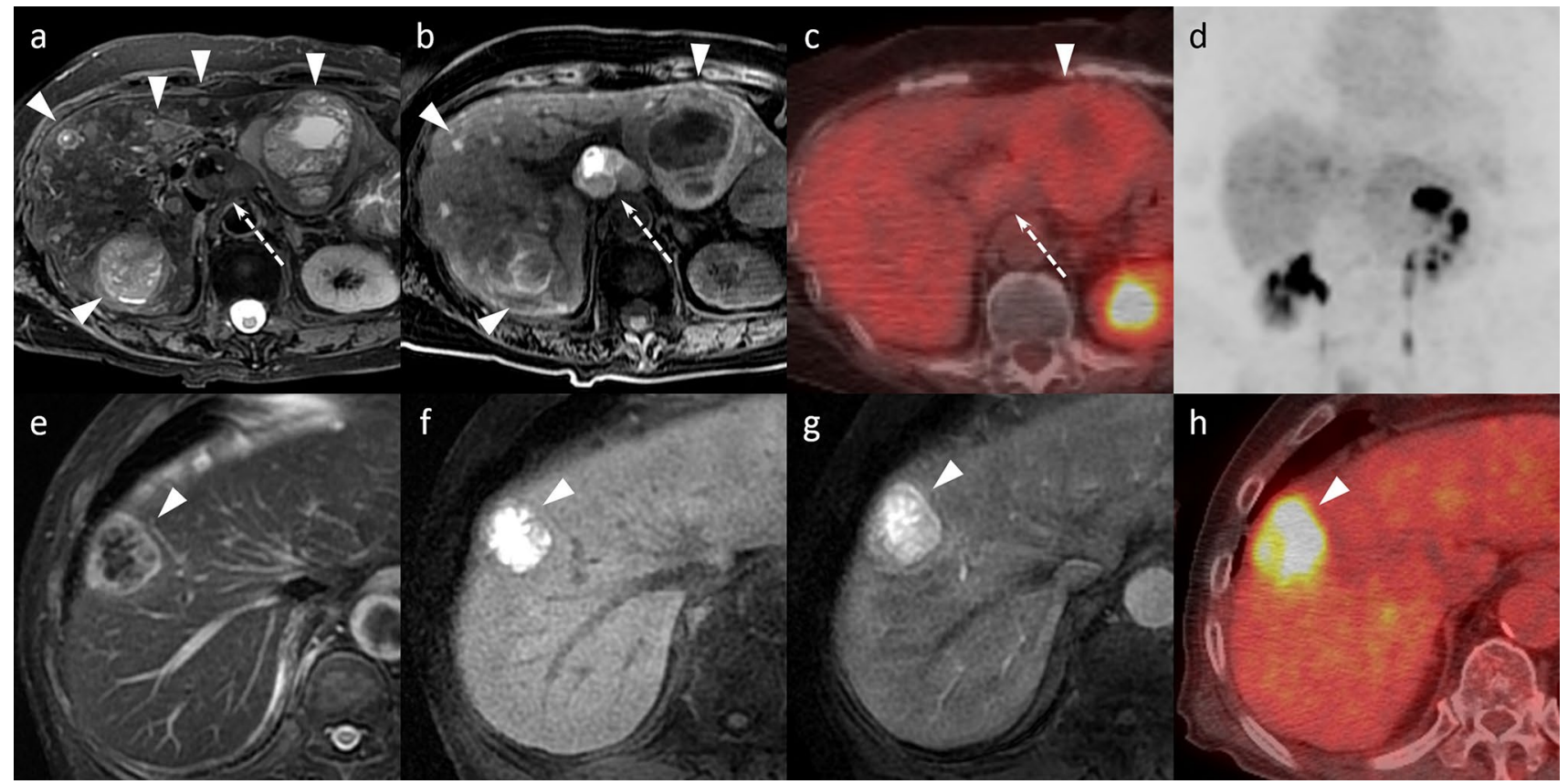

Fig. 12 Hepatic metastasis of uveal melanoma in a 71-year-old man (a-d) and in a 68-year-old man (e-h). a Fat-suppressed T2-weighted MRI shows multiple hyperintense masses (arrowheads) and a hypointense lymph node (arrow) in the hepatic portal region. b On fat-suppressed T1-weighted MRI, the masses appear mixed hyperand hypointense (arrowheads) and the lymph node is hyperintense (arrow). c No FDG uptake is detected in the masses (arrowhead) or lymph node (arrow) on PET/CT. d No FDG uptake is detected in the

and HCC. FDG imaging can differentiate atypical sclerosed hemangioma, which shows poor accumulation of FDG, from malignant tumor [90] (Fig. 14).

\section{Focal nodular hyperplasia}

Focal nodular hyperplasia (FNH) is the second most common benign hepatic tumor and is believed to be the result of a hyperplastic response of hepatocytes to the presence of a preexisting vascular malformation.

On contrast-enhanced CT and MRI, FNH shows homogenous hyperenhancement in the arterial and portal venous phases, and isoenhancement in the delayed or equilibrium phases. As FNH is usually treated conservatively, it must be distinguished from HCC. The majority of FNHs show iso- or hyperintensity relative to surrounding liver in the hepatobiliary phase of EOB-MRI [71, 99], and the overall diagnostic performance of hepatobiliary phase imaging in differentiating benign from malignant lesions is reported as having sensitivity of $96.6 \%$, specificity $87.6 \%$, and positive predictive value of $85 \%$ [93].

FDG-PET/CT has uncertain value in the imaging diagnosis of FNH, which commonly exhibits a similar (or even masses or the lymph node on coronal MIP PET. e Fat-suppressed T2-weighted MRI reveals a mass of hypointensity with hyperintense with rim (arrowhead). f The mass is hyperintense on fat-suppressed T1-weighted MRI (arrowhead). g The mass is also hyperintense on arterial phase MRI (arrowhead). h On PET/CT, the mass shows moderate FDG uptake (SUVmax 11.1) (arrowhead). Sixty percent of hepatic metastases of uveal melanoma show no uptake of FDG, the reason for which is unclear

lower) FDG uptake and retention pattern compared with normal liver tissue as they share the same metabolic mechanism $[85,100]$. It is of note that FNH is a hypervascular lesion that does not have accelerated glucose metabolism.

\section{Angiomyolipoma}

Angiomyolipoma (AML)/primary hepatic perivascular epithelioid cell tumor is a benign mesenchymal tumor composed of blood vessels, smooth muscle cells, and a varying amount of fat. AML containing a large amount of fat can be easily diagnosed on CT and MRI, but the imaging diagnosis is challenging if the fat component is relatively small. AML is often hypervascular in the arterial phase and shows washout, mimicking HCC. The imaging findings of presence of early draining vein and the absence of tumor capsule are useful for differentiating AML from HCC.

FDG accumulation in AML is similar to that in the normal part of the liver (Fig. 15) [101, 102], although the mechanism has not been elucidated. It is important to consider the possibility of hepatic AML exhibiting FDG uptake [103]. 


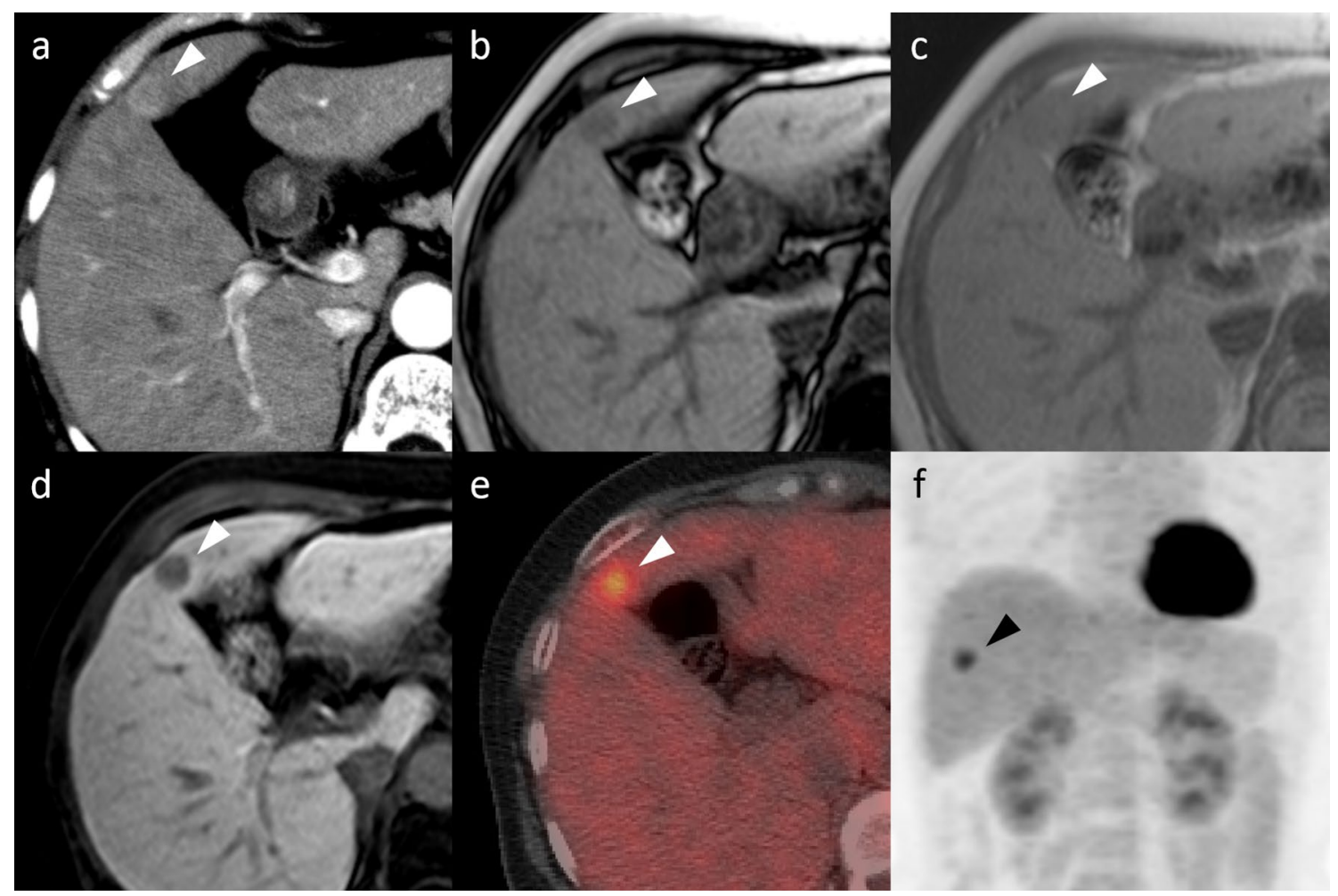

Fig. 13 Hepatocyte nuclear factor (HNF)-1 $\alpha$-inactivated hepatocellular adenoma (HCA) in a 67-year-old woman. a An incidentally detected nodule is hypervascular on arterial phase CT (arrowhead). b, c A fat deposit is observed within the mass on opposed-phase (b) and in-phase (c) MRI (arrowheads). d The nodule shows hypointensity on hepatobiliary phase MRI (arrowhead). e On PET/CT, the nodule shows obvious FDG uptake (SUVmax 5.2) (arrowhead). f The nodule shows obvious FDG uptake on coronal MIP PET (arrowhead). The diagnosis of HNF-1 $\alpha$-inactivated HCA was confirmed by surgical resection

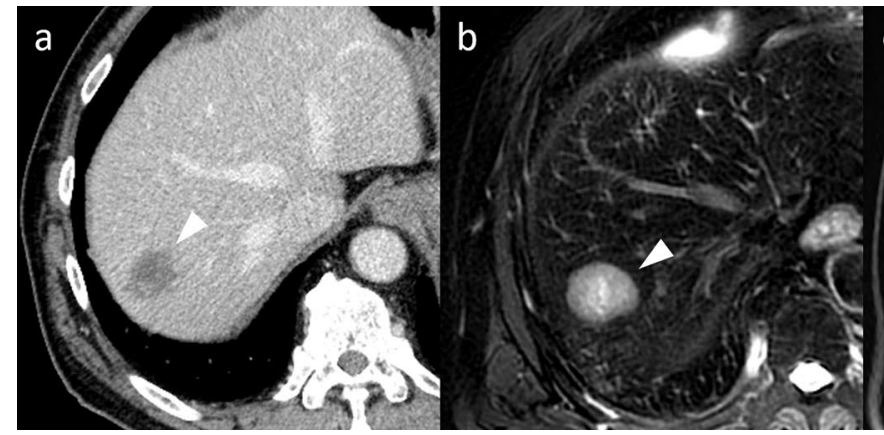

Fig. 14 Sclerosed cavernous hemangioma in a 72-year-old man. a An incidentally detected hepatic nodule (arrowhead) is hypovascular on equilibrium phase CT. b On fat-suppressed T2-weighted MRI, the nodule (arrowhead) is hyperintense to background liver and is

\section{Inflammatory pseudotumor}

Inflammatory pseudotumor is a rare condition that can present anywhere in the body, including the liver. Its incidence in the liver is reported to be $\sim 0.7 \%$ of all focal liver lesions, and is characterized by chronic infiltration of

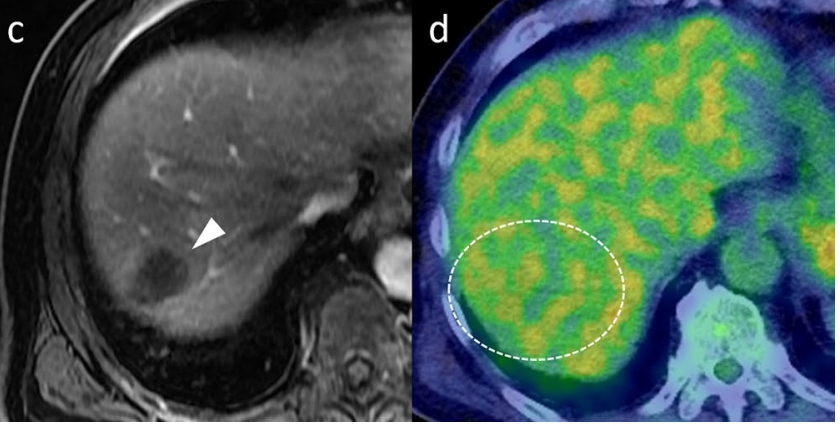

hypointense to cerebrospinal fluid. c No enhancement is observed on arterial phase MRI (arrowhead). d PET/CT shows similar uptake in the mass to background liver (dotted circle). The diagnosis of sclerosed hemangioma was confirmed by percutaneous liver biopsy

inflammatory cells and an area of fibrosis that can mimic malignant tumor. The etiology and pathogenesis remain unknown. Biologically, it has nonspecific symptoms and laboratory abnormalities such as fever, abdominal pain, abdominal discomfort, and leukocytosis. The radiological findings of ultrasound, CT, and MRI are also nonspecific, and it can be difficult to differentiate this pseudotumor 

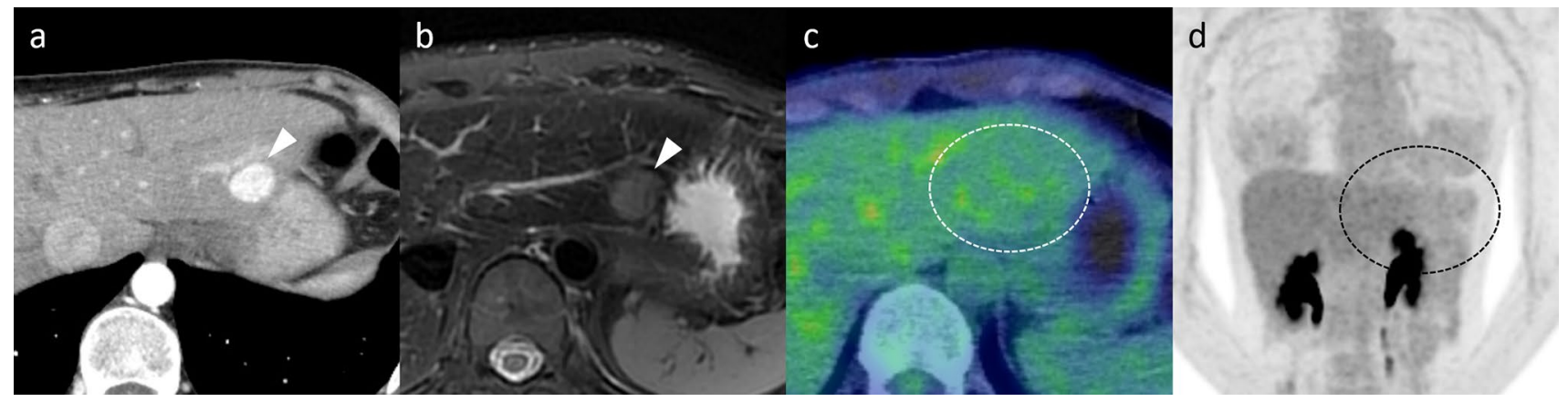

Fig. 15 Angiomyolipoma in a 34-year-old woman. a An incidentally detected hepatic nodule (arrowhead) is hypervascular, and no focal fat deposits are observed within the nodule on arterial phase CT. b The nodule (arrowhead) shows slight hyperintensity on fat-suppressed
T2-weighted MRI. c PET/CT shows no FDG uptake in the mass (dotted circle). d Coronal MIP PET also shows no FDG uptake in the mass (dotted circle). The diagnosis of angiomyolipoma was confirmed by surgical resection

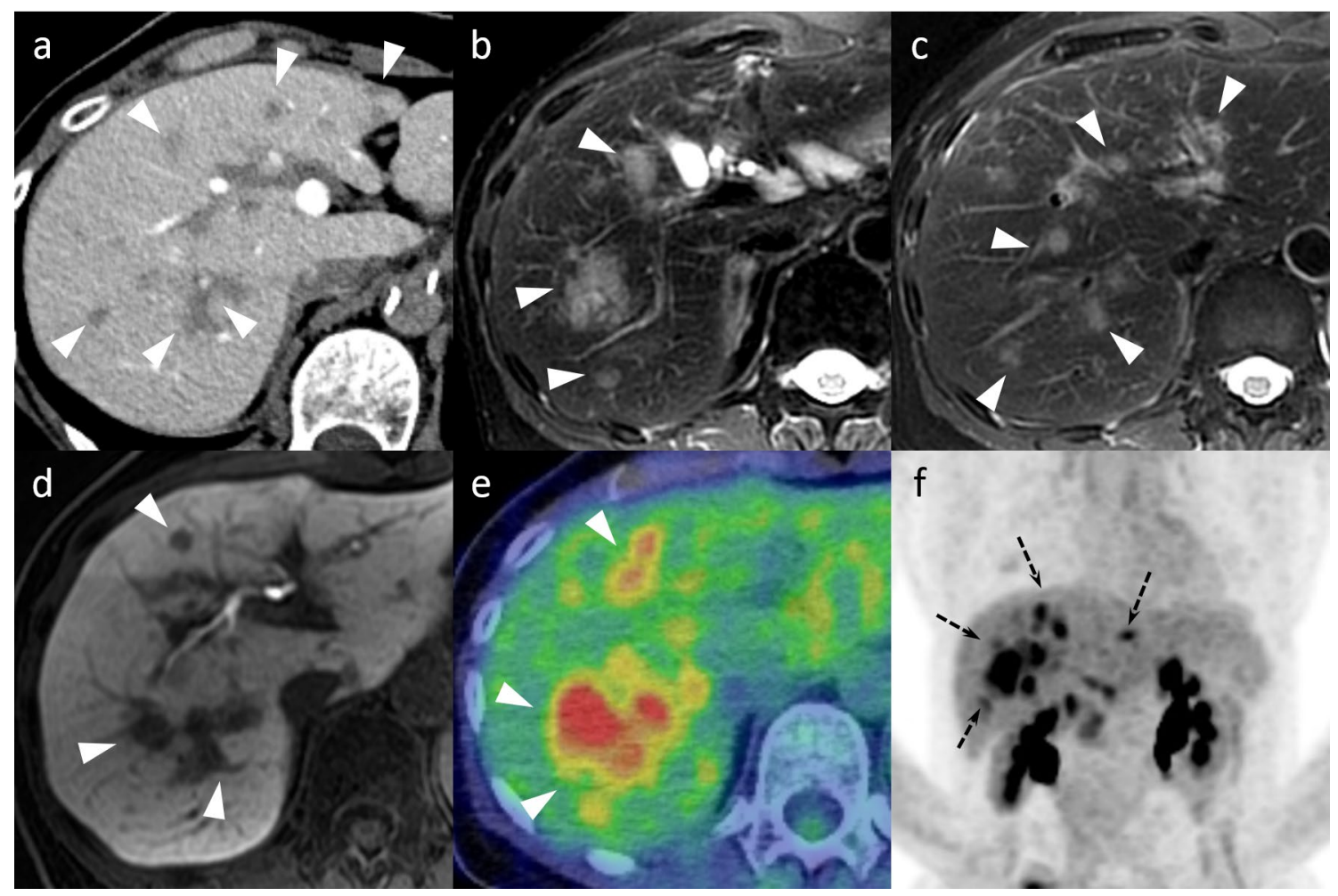

Fig. 16 Inflammatory pseudotumors in a 62-year-old man. a Portal phase CT reveals multiple hypovascular masses (arrowheads). b The masses (arrowheads) show ill-defined hyperintensity on fat-suppressed T2-weighted MRI. c Another slice position with the same sequence shows multiple ill-defined hyperintense masses of various sizes (arrowheads). d The masses show hypointensity on hepatobil- iary phase MRI (arrowheads). e The masses show high FDG uptake on PET/CT (arrowheads). f On coronal MIP PET, the masses show high FDG uptake (arrows). The diagnosis of inflammatory pseudotumors was confirmed by percutaneous liver biopsy, and the masses disappeared without treatment after 3 months from hepatic space-occupying lesions such as HCC, ICC, metastasis, and hepatic abscess. Furthermore, it is striking that this pseudotumor shows high FDG uptake [104, 105] (Fig. 16), which increases the difficulty of distinguishing it from malignancy.

\section{Pseudolymphoma}

Pseudolymphoma, also termed "reactive lymphoid hyperplasia" or "nodular lymphoid lesion", is a benign nodular 


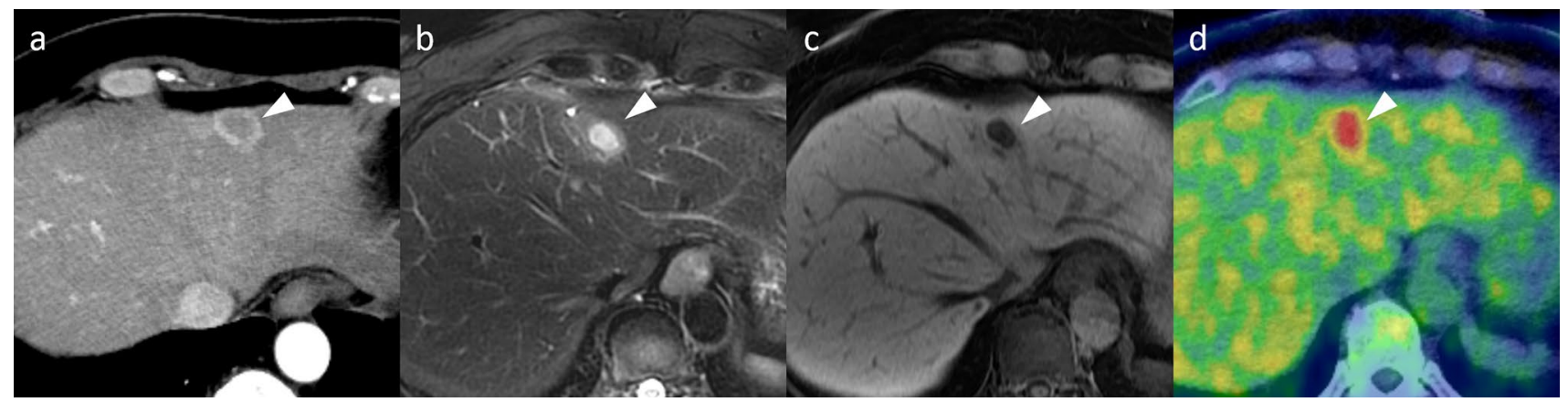

Fig. 17 Pseudolymphoma in a 44-year-old woman. a An incidentally detected hepatic nodule is hypovascular with perinodular enhancement on arterial phase CT (arrowhead). b The nodule shows hyperintensity on fat-suppressed T2-weighted MRI (arrowhead). c The nodule shows hypointensity on hepatobiliary phase MRI (arrowhead). d The nodule shows obvious uptake of FDG on PET/CT (SUVmax 12.8) (arrowhead). The diagnosis of pseudolymphoma was confirmed by percutaneous liver biopsy

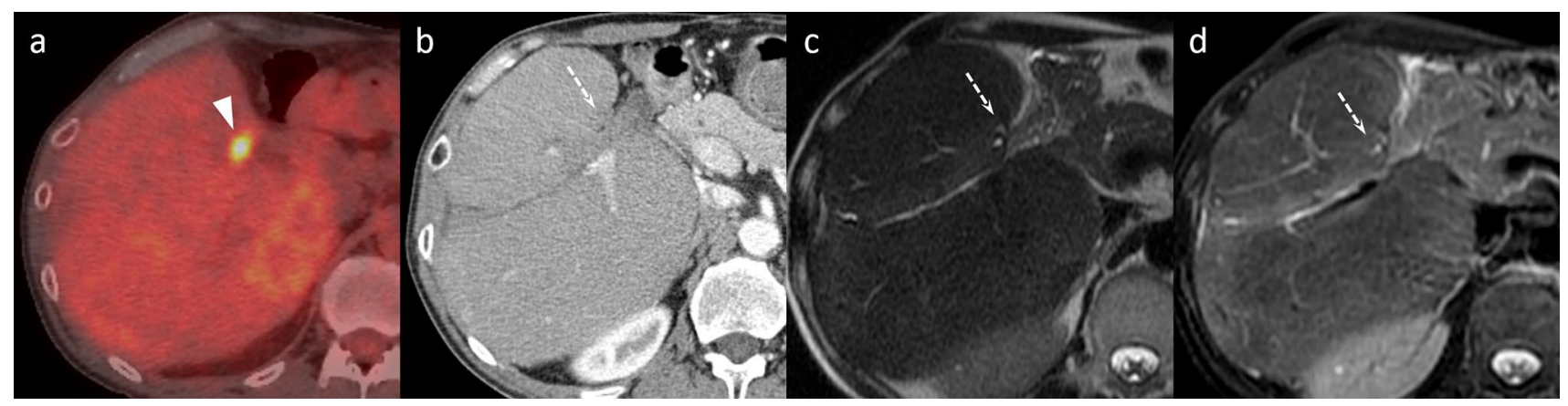

Fig. 18 Intrahepatic cholangitis in 56-year-old man with a history of intrahepatic cholangiocarcinoma. a PET/CT shows nodular uptake of FDG (arrowhead) that is suggestive of recurrence. b No lesion corresponding with the area of FDG uptake (arrow) is visualized on arterial phase CT. c T2-weighted MRI is also unable to depict a corresponding lesion (arrow). d Arterial phase MRI is also unable to depict a corresponding lesion (arrow)
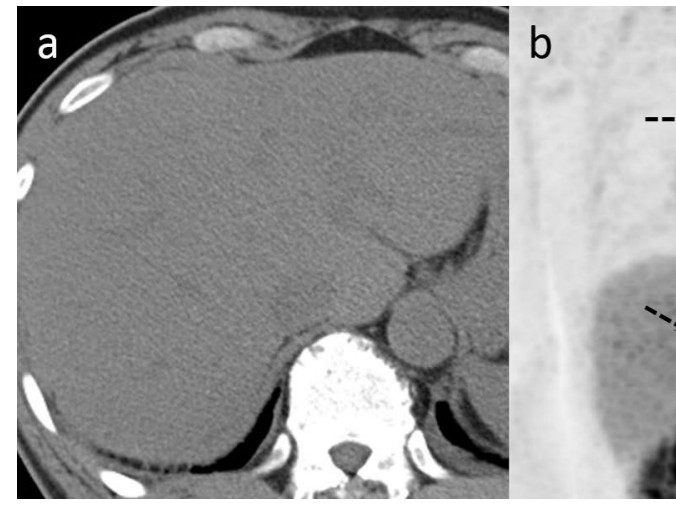

Fig. 19 Benign lymphoproliferative disorder with unknown etiology in 59-year-old man with pyrexia of unknown origin. a A hepatic lesion is not apparent on unenhanced CT. b Coronal MIP PET depicts hepatic and mediastinal lesions (arrows). Biopsy by mediastinoscopy could not obtain an adequate sample of any of the mediastinal lesions. c PET/CT reveals high FDG uptake (SUVmax 8.2) in the region of hepatic involvement (arrowhead) and was used to identify the biopsy site. The diagnosis of lymphoproliferative disorder of unknown etiology (not IgG4-related disease or malignant lymphoma) was confirmed by percutaneous liver biopsy 
lesion characterized histopathologically by marked proliferation of nonneoplastic, polyclonal lymphocytes that form follicles with an active germinal center [106]. It can occur in various organs, including the skin, orbit, lung, and gastrointestinal tract, but is rare in the liver, where it occurs predominantly in middle-aged females with hepatic tumor who also have autoimmune disease, malignant tumors, or chronic liver disease.

The preoperative diagnosis of hepatic pseudolymphoma is difficult because the findings of noninvasive radiological examinations such as ultrasonography, CT, and MRI are usually equivocal and have few specific features that can differentiate it from other malignant hepatic tumors. However, the lesions show FDG uptake (Fig. 17) (median SUV max 4.3; range 3.4-7.2) [107, 108].

\section{Inflammation and infection}

The broad spectrum of inflammatory and infectious liver diseases includes hepatic abscess, intrahepatic cholangitis, lymphoproliferative disorders including immunoglobulin
G4-related systemic disease, sarcoidosis; and tuberculosis, fungal, and parasitic infection [109].

FDG-PET/CT also has clinical utility in imaging suspected infection and inflammation (Fig. 18) to identify the source, map the extent and severity of disease, identify sites for tissue sampling (Fig. 19), and assess therapeutic response [86, 110], although its application for this purpose is controversial. FDG uptake in nonmalignant inflammatory conditions can produce false-positive results, particularly in patients with known or suspected malignancy [111], and correlation with the CT and MRI findings is important to avoid misdiagnosis.

Increased FDG uptake in inflammation and inflammation is due, at least in part, to an increase in GLUT, as with malignant cells [112]. Activated inflammatory cells also demonstrate overexpression of GLUT-1 and/or GLUT-3 [113-115]. In addition, in inflammatory conditions, the affinity of GLUT for deoxyglucose is apparently increased by various cytokines and growth factors, a phenomenon that has not been observed in tumors [116].

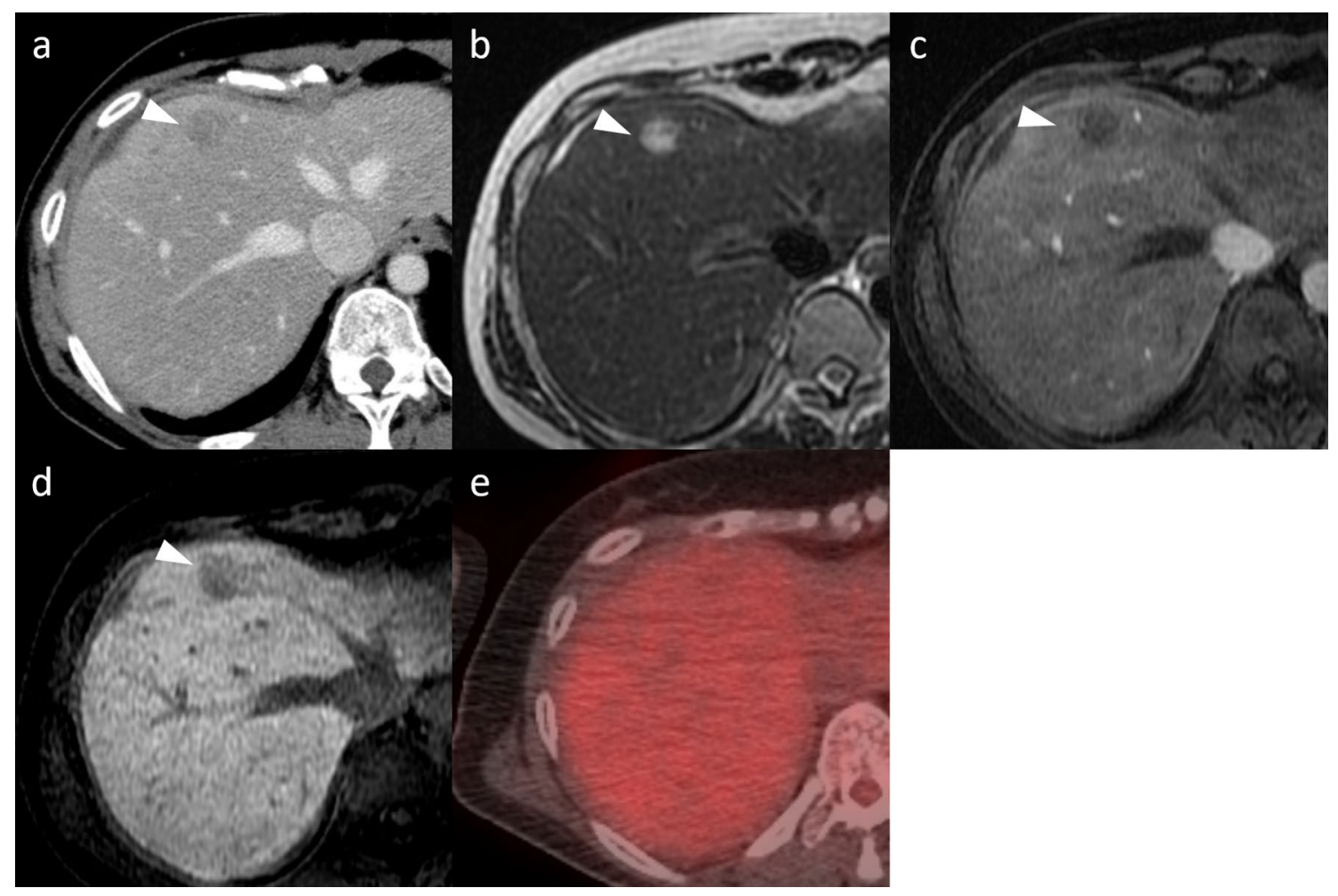

Fig. 20 Focal sinusoidal obstruction syndrome during chemotherapy (oxaliplatin + TS-1) in a 41-year-old woman with a history of rectal adenocarcinoma. a Arterial phase CT reveals a new hypovascular nodule (arrowhead) in the medial segment of the liver. b The nodule shows hyperintensity on T2-weighted MRI (arrowhead). c The nodule is hypovascular to normal liver on the arterial phase of dynamic contrast MRI (arrowhead). d The nodule shows hypointensity in the hepatobiliary phase (arrowhead). These findings are slightly different from those of metastasis, but could not exclude metastasis. e The lack of FDG uptake on PET/CT indicates focal sinusoidal obstruction syndrome rather than metastasis, and liver biopsy confirmed the diagnosis of sinusoidal obstruction syndrome 


\section{Focal sinusoidal obstruction syndrome}

Sinusoidal obstruction syndrome (SOS), also termed toxic sinusoidal injury, veno-occlusive disease, or "blue liver syndrome", is a commonly seen vascular pattern of drug-induced liver injury that has been associated most frequently with oxaliplatin-based chemotherapy, which is mainly used in gastrointestinal malignancy [117].

SOS is characterized by the histologic findings of sinusoidal dilatation and congestion, centrilobular vein fibrosis and obstruction, and perisinusoidal fibrosis. These changes are generally distributed irregularly and diffusely within the whole hepatic parenchyma, and have the highly specific imaging appearance of diffuse and reticular hypointensity on hepatobiliary phase EOB-enhanced MRI [118]. SOS has no glycometabolism and thus shows no uptake of FDG, which is obvious considering the pathological findings. Uncommonly, SOS appears as a nodular lesion $[119,120]$, in which case FDG-PET/CT imaging is useful to distinguish focal SOS from hepatic metastases combined with $\mathrm{CT}$ and MR images (Fig. 20), especially in patients who are contraindicated for percutaneous liver biopsy.

\section{Intratumoral hemorrhage in the acute phase}

Increased FDG uptake in response to hemorrhage has been reported in numerous diseases, including hepatic tumor, adrenal hemorrhage, peri-renal hemorrhage, aortic wall hematoma, pelvic hematoma, and subarachnoid hemorrhage. In the acute phase, FDG uptake in the hematoma is due to extravasation of activated macrophages; however, uptake weakens in the healing phase, suggesting that the procallus itself is associated with elevated glycolytic metabolism.

Because the accumulation of FDG due to intratumoral hemorrhage could occur in any hepatic tumor, it is important to review the FDG-PET/CT imaging findings along with conventional $\mathrm{CT}$ and MRI to prevent a misdiagnosis.

\section{Conclusion}

FDG-PET/CT imaging has proved invaluable in diagnosis, detection, assessment of differentiation, staging, response to treatment, and prediction of prognosis in a wide variety of hepatic lesions. Knowledge of the degree of FDG uptake in each lesion type is essential for accurate image interpretation and to enable selection of an appropriate therapeutic strategy. An understanding of the underlying molecular background is also valuable in this regard.
Funding This study received no specific grant from any funding agency.

\section{Compliance with ethical standards}

Conflict of interest The authors declare that they have no conflicts of interest.

Ethical approval All procedures performed in studies involving human participants were in accordance with the ethical standards of the institutional and/or national research committee and with the 1964 Helsinki Declaration and its later amendments or comparable ethical standards.

Informed consent The requirement for written informed consent was waived for this type of review article based on the opt-out system of our institution.

\section{References}

1. Raddatz D, Ramadori G. Carbohydrate metabolism and the liver: actual aspects from physiology and disease. Z Gastroenterol. 2007;45(1):51-62.

2. Mueckler M, Thorens B. The SLC2 (GLUT) family of membrane transporters. Mol Aspects Med. 2013;34(2-3):121-38.

3. Wilson JE. Hexokinases. Rev Physiol Biochem Pharmacol. 1995;126:65-198.

4. van Schaftingen E, Gerin I. The glucose-6-phosphatase system. Biochem J. 2002;362(Pt 3):513-32.

5. Burchell A. Molecular pathology of glucose-6-phosphatase. FASEB J. 1990;4(12):2978-88.

6. Pauwels EK, Ribeiro MJ, Stoot JH, McCready VR, Bourguignon M, Maziere B. FDG accumulation and tumor biology. Nucl Med Biol. 1998;25(4):317-22.

7. Jadvar H, Alavi A, Gambhir SS. 18F-FDG uptake in lung, breast, and colon cancers: molecular biology correlates and disease characterization. J Nucl Med. 2009;50(11):1820-7.

8. Kim BT, Kim Y, Lee KS, Yoon SB, Cheon EM, Kwon OJ, et al. Localized form of bronchioloalveolar carcinoma: FDG PET findings. AJR Am J Roentgenol. 1998;170(4):935-9.

9. Jadvar H, Segall GM. False-negative fluorine-18-FDG PET in metastatic carcinoid. J Nucl Med. 1997;38(9):1382-3.

10. Schuurbiers OC, Meijer TW, Kaanders JH, Looijen-Salamon MG, de Geus-Oei LF, van der Drift MA, et al. Glucose metabolism in NSCLC is histology-specific and diverges the prognostic potential of 18FDG-PET for adenocarcinoma and squamous cell carcinoma. J Thorac Oncol. 2014;9(10):1485-93.

11. Blake MA, Singh A, Setty BN, Slattery J, Kalra M, Maher $\mathrm{MM}$, et al. Pearls and pitfalls in interpretation of abdominal and pelvic PET-CT. RadioGraphics. 2006;26(5):1335-533.

12. Sureshbabu W, Mawlawi O. PET/CT imaging artifacts. J Nucl Med Technol. 2005;33(3):156-61.

13. Dizendorf E, Hany TF, Buck A, Von Schulthess GK, Burger C. Cause and magnitude of the error induced by oral CT contrast agent in CT-based attenuation correction of PET emission studies. J Nucl Med. 2003;44(5):732-8.

14. Goerres GW, Ziegler SI, Burger C, Berthold T, Von Schulthess GK, Buck A. Artifacts at PET and PET/CT caused by metallic hip prosthetic material. Radiology. 2003;226(2):577-84.

15. Goerres GW, Burger C, Kamel E, Seifert B, Kaim AH, Buck A, et al. Respiration-induced attenuation artifact at PET/CT: technical considerations. Radiology. 2003;226(3):906-10. 
16. Nakamoto Y, Tatsumi M, Cohade C, Osman M, Marshall LT, Wahl RL. Accuracy of image fusion of normal upper abdominal organs visualized with PET/CT. Eur J Nucl Med Mol Imaging. 2003;30(4):597-602.

17. Vogel WV, van Dalen JA, Wiering B, Huisman H, Corstens FH, Ruers TJ, et al. Evaluation of image registration in PET/CT of the liver and recommendations for optimized imaging. J Nucl Med. 2007;48(6):910-9.

18. Soret M, Bacharach SL, Buvat I. Partial-volume effect in PET tumor imaging. J Nucl Med. 2007;48(6):932-45.

19. Fong Y, Saldinger PF, Akhurst T, Macapinlac H, Yeung H, Finn RD, et al. Utility of 18F-FDG positron emission tomography scanning in selection of patients for resection of hepatic colorectal metastases. Am J Surg. 1999;178(4):282-7.

20. Delbeke D, Vitola JV, Sandler MP, Arildsen RC, Powers TA, Wright JK Jr, et al. Staging recurrent metastatic colorectal carcinoma with PET. J Nucl Med. 1997;38(8):1196-201.

21. Paquet N, Albert A, Foidart J, Hustinx R. Within-patient variability of (18)F-FDG: standardized uptake values in normal tissues. J Nucl Med. 2004;45(5):784-8.

22. Keramida G, Dizdarevic S, Bush J, Peters AM. Quantification of tumour (18) F-FDG uptake: Normalise to blood glucose or scale to liver uptake? Eur Radiol. 2015;25(9):2701-8.

23. Bu"sing KA, Scho"nberg SO, Brade J, Wasser K. Impact of blood glucose, diabetes, insulin, and obesity on standardized uptake values in tumors and healthy organs on 18 F-FDG PET/ CT. Nucl Med Biol. 2013;40(2):206-13.

24. Viglianti BL, Wong KK, Wimer SM, Parameswaran A, Nan $\mathrm{B}, \mathrm{Ky} \mathrm{C}$, et al. Effect of hyperglycemia on brain and liver 18F-FDG standardized uptake value (FDG SUV) measured by quantitative positron emission tomography (PET) imaging. Biomed Pharmacother. 2017;88:1038-45.

25. Mahmud MH, Nordin AJ, Ahmad Saad FF, Azman AZ. Impacts of biological and procedural factors on semiquantification uptake value of liver in fluorine-18 fluorodeoxyglucose positron emission tomography/computed tomography imaging. Quant Imaging Med Surg. 2015;5(5):700-7.

26. Liu Y. Fluorodeoxyglucose uptake in absence of CT abnormality on PET-CT: What is it? World J Radiol. 2013;5(12):460-7.

27. Torizuka T, Tamaki N, Inokuma T, Magata Y, Sasayama S, Yonekura Y, et al. In vivo assessment of glucose metabolism in hepatocellular carcinoma with FDG-PET. J Nucl Med. 1995;36(10):1811-7.

28. Wudel LJ Jr, Delbeke D, Morris D, Rice M, Washington MK, Shyr Y, et al. The role of [18F]fluorodeoxyglucose positron emission tomography imaging in the evaluation of hepatocellular carcinoma. Am Surg. 2003;69(2):117-26.

29. Cronin CG, Swords R, Truong MT, Viswanathan C, Rohren E, Giles FJ, et al. Clinical utility of PET/CT in lymphoma. AJR Am J Roentgenol. 2010;194(1):W91-W103.

30. Weiler-Sagie M, Bushelev O, Epelbaum R, Dann EJ, Haim N, Avivi I, et al. (18)F-FDG avidity in lymphoma readdressed: a study of 766 patients. J Nucl Med. 2010;51(1):25-30.

31. Binderup T, Knigge U, Loft A, Mortensen J, Pfeifer A, Federspiel B, et al. Functional imaging of neuroendocrine tumors: a head-to-head comparison of somatostatin receptor scintigraphy, 123I-MIBG scintigraphy, and 18F-FDG PET. J Nucl Med. 201;51(5):704-12.

32. Garin E, Le Jeune F, Devillers A, Cuggia M, de Lajarte-Thirouard AS, Bouriel C, et al. Predictive value of 18F-FDG PET and somatostatin receptor scintigraphy in patients with metastatic endocrine tumors. J Nucl Med. 2009;50(6):858-64.

33. Lee SD, Kim SH, Kim YK, Kim C, Kim SK, Han SS, et al. (18) F-FDG-PET/CT predicts early tumor recurrence in living donor liver transplantation for hepatocellular carcinoma. Transpl Int. 2013;26(1):50-60.
34. Huang WY, Kao CH, Huang WS, Chen CM, Chang LP, Lee MS, et al. 18F-FDG PET and combined 18F-FDG-contrast CT parameters as predictors of tumor control for hepatocellular carcinoma after stereotactic ablative radiotherapy. J Nucl Med. 2013;54(10):1710-6.

35. Song MJ, Bae SH, Lee SW, Song DS, Kim HY, Yoo IeR, et al. 18 F-fluorodeoxyglucose PET/CT predicts tumour progression after transarterial chemoembolization in hepatocellular carcinoma. Eur J Nucl Med Mol Imaging. 2013;40(6):865-73.

36. Sun DW, An L, Wei F, Mu L, Shi XJ, Wang CL, et al. Prognostic significance of parameters from pretreatment (18)F-FDG PET in hepatocellular carcinoma: a meta-analysis. Abdom Radiol (NY). 2016;41(1):33-41.

37. Binderup T, Knigge U, Loft A, Federspiel B, Kjaer A. 18F-fluorodeoxyglucose positron emission tomography predicts survival of patients with neuroendocrine tumors. Clin Cancer Res. 2010;16(3):978-85.

38. Lee JD, Yang WI, Park YN, Kim KS, Choi JS, Yun M, et al. Different glucose uptake and glycolytic mechanisms between hepatocellular carcinoma and intrahepatic mass-forming cholangiocarcinoma with increased (18) F-FDG uptake. J Nucl Med. 2005;46(10):1753-9.

39. Kawaoka T, Aikata H, Takaki S, Uka K, Azakami T, Saneto H, et al. FDG positron emission tomography/computed tomography for the detection of extrahepatic metastases from hepatocellular carcinoma. Hepatol Res. 2009;39(2):134-42.

40. Anderson CD, Rice MH, Pinson CW, Chapman WC, Chari RS, Delbeke D. Fluorodeoxyglucose PET imaging in the evaluation of gallbladder carcinoma and cholangiocarcinoma. J Gastrointest Surg. 2004;8(1):90-7.

41. Kim YJ, Yun M, Lee WJ, Kim KS, Lee JD. Usefulness of 18FFDG PET in intrahepatic cholangiocarcinoma. Eur J Nucl Med Mol Imaging. 2003;30(11):1467-72.

42. Suzuki H, Komuta M, Bolog A, Yokobori T, Wada S, Araki $\mathrm{K}$, et al. Relationship between 18-F-fluoro-deoxy-D-glucose uptake and expression of glucose transporter 1 and pyruv-ate kinase M2 in intrahepatic cholangiocarcinoma. Dig Liver Dis. 2015;47(7):590-6.

43. Jadvar H, Henderson RW, Conti PS. [F-18] fluorodeoxyglucose positron emission tomography and positron emission tomography: computed tomography in recurrent and metastatic cholangiocarcinoma. J Comput Assist Tomogr. 2007;31(2):223-8.

44. Corvera CU, Blumgart LH, Akhurst T, DeMatteo RP, D'Angelica $\mathrm{M}$, Fong Y, et al. 18F-fluorodeoxyglucose positron emission tomography influences management decisions in patients with biliary cancer. J Am Coll Surg. 2008;206(1):57-655.

45. Paradis V, Fukayama M, Park YN, Schirmacher, P. Tumors of the liver and intrahepatic bile ducts. In: WHO classification of tumours editorial board. WHO classification of tumours of the digestive system. 5th ed. Lyon: IARC; 2019. pp. 215-64.

46. Mori N, Ichikawa T, Hashimoto J, Yamashita T, Yamada M, Hirabayashi K, et al. Cholangiolocellular carcinoma of the liver exhibiting high F-18 FDG uptake. Tokai J Exp Clin Med. 2016;41(2):60-4.

47. Lim CH, Moon SH, Cho YS, Choi JY, Lee KH, Hyun SH. Prognostic value of $18 \mathrm{~F}$-fluorodeoxyglucose positron emission tomography/computed tomography in patients with combined hepatocellular-cholangiocarcinoma. Eur J Nucl Med Mol Imaging. 2019;46(8):1705-12.

48. Yi LL, Zhang JX, Zhou SG, Wang J, Huang YQ, Li J, et al. $\mathrm{CT}$ and MRI studies of hepatic angiosarcoma. Clin Radiol. 2019;74(5):406.e1-.e8.

49. Kamatani T, Iguchi H, Okada T, Yamazaki H, Tsunoda H, Watanabe M, et al. Co-registered positron emission tomography/computed tomography and gadolinium-ethoxybenzyldiethylenetriamine pentaacetic acid magnetic resonance imaging 
features of multiple angiosarcoma of the liver. Hepatol Res. 2014;44(10):E297-303.

50. Maeda T, Tateishi U, Hasegawa T, Ojima H, Arai Y, Sugimura K. Primary hepatic angiosarcoma on coregistered FDG PET and CT images. AJR Am J Roentgenol. 2007;188(6):1615-7.

51. Mark L, Delmore F, Creech JL Jr, Ogden LL II, Fadell EH, Songster CL, et al. Clinical and morphologic features of hepatic angiosarcoma in vinyl chloride workers. Cancer. 1976;37(1):149-63.

52. Avlonitis VS, Linos D. Primary hepatic lymphoma: a review. Eur J Surg. 1999;165(8):725-9.

53. Ohsawa M, Aozasa K, Horiuchi K, Kataoka M, Hida J, Shimada $\mathrm{H}$, et al. Malignant lymphoma of the liver. Report of five cases and review of the literature. Dig Dis Sci. 1992;37(7):1105-9.

54. Lee JA, Jeong WK, Min JH, Kim J. Primary hepatic lymphoma mimicking acute hepatitis. Clin Mol Hepatol. 2013;19(3):320-3.

55. Juweid ME, Cheson BD. Role of positron emission tomography in lymphoma. J Clin Oncol. 2005;23(21):4577-80.

56. Noy A, Schöder H, Gönen M, Weissler M, Ertelt K, Cohler C, et al. The majority of transformed lymphomas have high standardized uptake values (SUVs) on positron emission tomography (PET) scanning similar to diffuse large B-cell lymphoma (DLBCL). Ann Oncol. 2009;20(3):508-12.

57. Rodriguez M, Rehn S, Ahlström H, Sundström C, Glimelius B. Predicting malignancy grade with PET in non-Hodgkin's lymphoma. J Nucl Med. 1995;36(10):1790-6.

58. Tsimberidou AM, Wierda WG, Plunkett W, Kurzrock R, O'Brien S, Wen S, et al. Phase I-II study of oxaliplatin, fludarabine, cytarabine, and rituximab combination therapy in patients with Richter's syndrome or fludarabine-refractory chronic lymphocytic leukemia. J Clin Oncol. 2008;26(2):196-203.

59. Liu YM, Zhai XM, Wu YW. Biological correlation between glucose transporters, Ki-67 and 2-deoxy-2-[18F]-fluoro-D-glucose uptake in diffuse large B-cell lymphoma and natural killer/T-cell lymphoma. Genet Mol Res. 2016;15(2).

60. Hirose Y, Suefuji H, Kaida H, Hayakawa M, Hattori S, Kurata S, et al. Relationship between 2-deoxy-2-[(18)F]-fluoro-D-glucose uptake and clinicopathological factors in patients with diffuse large B-cell lymphoma. Leuk Lymphoma. 2014;55(3):520-5.

61. Shim HK, Lee WW, Park SY, Kim H, Kim SE. Relationship between FDG uptake and expressions of glucose transporter type 1, type 3, and hexokinase-II in Reed-Sternberg cells of Hodgkin lymphoma. Oncol Res. 2009;17(7):331-7.

62. Lloyd RV, Osamura RY, Kloppel G, Rosai J, editors. WHO Classification of Tumours of Endocrine Organs. 4th ed. IARC: Lyon; 2017.

63. Benzerdjeb N, Berna P, Sevestre H. GLUT1: A novel tool reflecting proliferative activity of lung neuroendocrine tumors? Pathol Int. 2017;67(1):32-6.

64. D'souza MM, Sharma R, Mondal A, Jaimini A, Tripathi M, Saw SK, et al. Prospective evaluation of CECT and 18F-FDG-PET/ $\mathrm{CT}$ in detection of hepatic metastases. Nucl Med Commun. 2009;30(2):117-25.

65. Arulampalam TH, Francis DL, Visvikis D, Taylor I, Ell PJ. FDGPET for the pre-operative evaluation of colorectal liver metastases. Eur J Surg Oncol. 2004;30(3):286-91.

66. Sivesgaard K, Larsen LP, Sørensen M, Kramer S, Schlander S, Amanavicius N, et al. Diagnostic accuracy of CE-CT, MRI and FDG PET/CT for detecting colorectal cancer liver metastases in patients considered eligible for hepatic resection and/or local ablation. Eur Radiol. 2018;28(11):4735-47.

67. Lincke T. Zech CJ. Liver metastases: Detection and staging. Eur J Radiol; 2017. p. 76-82.

68. Zimmerman RL, Fogt F, Burke M, Murakata LA. Assessment of Glut-1 expression in cholangiocarcinoma, benign biliary lesions and hepatocellular carcinoma. Oncol Rep Oncol Rep. 2002;9(4):689-92.
69. Pinilla I, Rodríguez-Vigil B, Gómez-León N. Integrated FDG PET/CT: Utility and Applications in Clinical Oncology. Clin Med Oncol. 2008;2:181-98.

70. Poeppel TD, Krause BJ, Heusner TA, Boy C, Bockisch A, Antoch G. PET/CT for the staging and follow-up of patients with malignancies. Eur J Radiol. 2009;70(3):382-92.

71. Wang Z, Chen JQ, Liu JL, Qin XG, Huang Y. FDG-PET in diagnosis, staging and prognosis of pancreatic carcinoma: a meta-analysis. World J Gastroenterol. 2013;19(29):4808-17.

72. Liu Y. Role of positron emission tomography with fluorodeoxyglucose in prostate cancer. Oncol Lett. 2014;7(6):2013-8.

73. Iwano S, Ito S, Tsuchiya K, Kato K, Naganawa S. What causes false-negative PET findings for solid-type lung cancer? Lung Cancer. 2013;79(2):132-6.

74. Higashi K, Clavo AC, Wahl RL. Does FDG uptake measure proliferative activity of human cancer cells? In vitro comparison with DNA flow cytometry and tritiated thymidine uptake. J Nucl Med. 1993;34(3):414-9.

75. Berger KL, Nicholson SA, Dehdashti F, Siegel BA. FDG PET evaluation of mucinous neoplasms: correlation of FDG uptake with histopathologic features. AJR Am J Roentgenol. 2000;174(4):1005-8.

76. Picchia S, Riddell A, Terlizzo M, Bali MA. Liver metastasis from adenoid cystic carcinoma: imaging and histologic features. Curr Probl Cancer. 2019;43(4):331-5.

77. Takahashi M, Kume H, Koyama K, Nakagawa T, Fujimura $\mathrm{T}$, Morikawa T, et al. Preoperative evaluation of renal cell carcinoma by using 18F-FDG PET/CT. Clin Nucl Med. 2015;40(12):936-40.

78. Ozerlat I. Kidney cancer: targeted therapy of glucose uptake via GLUT1 kills RCC cells. Nat Rev Urol. 2011;8(9):471.

79. Chan DA, Sutphin PD, Nguyen P, Turcotte S, Lai EW, Banh A, et al. Targeting GLUT1 and the Warburg effect in renal cell carcinoma by chemical synthetic lethality. Sci Transl Med. 2011;3(94):94ra70.

80. Schmoll D, Balabanov S, Schwarck D, Burchell A, Kleist B, Zimmermann U, et al. Differential expression of the subunits of the glucose-6-phosphatase system in the clear cell type of human renal cell carcinoma-no evidence for an overexpression of protein kinase B. Cancer Lett. 2001;167(1):85-90.

81. Macfarlane DJ, Sondak V, Johnson T, Wahl RL. Prospective evaluation of 2-fluorine-[18F]-fluoro-2-deoxy-D-glucose positron emission tomography in staging of regional lymph nodes in patients with cutaneous malignant melanoma. J Clin Oncol. 1998;16(5):1770-6.

82. Lucignani G, Paganelli G, Modorati G, Pieralli S, Rizzo G, Magnani P, et al. MRI, antibody-guided scintigraphy, and glucose metabolism in uveal melanoma. J Comput Assist Tomogr. 1992;16(1):77-83.

83. Spraul CW, Lang GE, Lang GK. Value of positron emission tomography in the diagnosis of malignant ocular tumors. Ophthalmologica. 2001;215(3):163-8.

84. Strobel K, Bode B, Dummer R, Veit-Haibach P, Fischer DR, Imhof $\mathrm{L}$, et al. Limited value of 18F-FDG PET/CT and S-100B tumour marker in the detection of liver metastases from uveal melanoma compared to liver metastases from cutaneous melanoma. Eur J Nucl Med Mol Imaging. 2009;36(11):1774-822.

85. Sacks A, Peller PJ, Surasi DS, Chatburn L, Mercier G, Subramaniam RM. Value of PET/CT in the management of primary hepatobiliary tumors, part 2. AJR Am J Roentgenol. 2011;197(2):W260-W265265.

86. Love C, Tomas MB, Tronco GG, Palestro CJ. FDG PET of infection and inflammation. Radiographics. 2005;25(5):1357-68.

87. Magini G, Farsad M, Frigerio M, Serra C, Colecchia A, Jovine E, et al. C-11 acetate does not enhance usefulness of F-18 
FDG PET/CT in differentiating between focal nodular hyperplasia and hepatic adenoma. Clin Nucl Med Clin Nucl Med. 2009;34(10):659-65.

88. Ozaki K, Harada K, Terayama N, Matsui O, Saitoh S, Tomimaru Y, et al. Hepatocyte nuclear factor $1 \alpha$-inactivated hepatocellular adenomas exhibit high (18)F-fludeoxyglucose uptake associated with glucose-6-phosphate transporter inactivation. Br J Radiol. 2016;89(1063):20160265.

89. Lee SY, Kingham TP, LaGratta MD, Jessurun J, Cherqui D, Jarnagin WR, et al. PET-avid hepatocellular adenomas: incidental findings associated with HNF1- $\alpha$ mutated lesions. HPB (Oxford). 2016;18(1):41-8.

90. Delbeke D, Martin WH, Sandler MP, Chapman WC, Wright JK Jr, Pinson CW. Evaluation of benign vs malignant hepatic lesions with positron emission tomography. Arch Surg 1998; 133:510-515, discussion 515-516

91. Nault JC, Paradis V, Cherqui D, Vilgrain V, Zucman-Rossi J. Molecular classification of hepatocellular adenoma in clinical practice. J Hepatol. 2017;67(5):1074-83.

92. McInnes MD, Hibbert RM, Inácio JR, Schieda N. Focal Nodular Hyperplasia and Hepatocellular Adenoma: Accuracy of Gadoxetic Acid-enhanced MR Imaging-A Systematic Review. Radiology. 2015;277(2):413-23.

93. Grazioli L, Bondioni MP, Haradome H, Motosugi U, Tinti R, Frittoli B, et al. Hepatocellular adenoma and focal nodular hyperplasia: value of gadoxetic acid-enhanced MR imaging in differential diagnosis. Radiology. 2012;262(2):520-9.

94. Kurtaran A, Becherer A, Pfeffel F, Müller C, Traub T, Schmaljohann J, et al. 18F-fluorodeoxyglucose (FDG)-PET features of focal nodular hyperplasia $(\mathrm{FNH})$ of the liver. Liver. 2000;20(6):487-90.

95. Rebouissou S, Imbeaud S, Balabaud C, Boulanger V, BertrandMichel J, Tercé F, et al. HNF1alpha inactivation promotes lipogenesis in human hepatocellular adenoma independently of SREBP-1 and carbohydrate-response element-binding protein (ChREBP) activation. J Biol Chem. 2007;282(19):14437-46.

96. Vilgrain V, Boulos L, Vullierme MP, Denys A, Terris B, Menu Y. Imaging of atypical hemangiomas of the liver with pathologic correlation. Radiographics. 2000;20(2):379-97.

97. Gibney RG, Hendin AP, Cooperberg PL. Sonographically detected hepatic hemangiomas: absence of change over time. AJR Am J Roentgenol. 1987;149(5):953-7.

98. Shimada K, Nakamoto Y, Isoda H, Saito H, Arizono S, Shibata $\mathrm{T}$, et al. FDG PET for giant cavernous hemangioma: important clue to differentiate from a malignant vascular tumor in the liver. Clin Nucl Med. 2010;35(12):924-6.

99. Mohajer K, Frydrychowicz A, Robbins JB, Loeffler AG, Reed TD, Reeder SB. Characterization of hepatic adenoma and focal nodular hyperplasia with gadoxetic acid. J Magn Reson Imaging. 2012;36(3):686-96.

100. Morana G, Grazioli L, Kirchin MA, Bondioni MP, Faccioli N, Guarise A, et al. Solid hypervascular liver lesions: accurate identification of true benign lesions on enhanced dynamic and hepatobiliary phase magnetic resonance imaging after gadobenate dimeglumine administration. Invest Radiol. 2011;46(4):225-39.

101. Wang X, Wang J, Cheng X, Li F, Huo L. Hepatic Angiomyolipoma Having FDG Uptake at the Similar Level of the Normal Liver Parenchyma. Clin Nucl Med. 2019;44(7):599-601.

102. Lee SJ, Kim SY, Kim KW, Shin YM, Kim HJ, Lee JS, et al. Hepatic angiomyolipoma with minimal fat, mimicking hepatocellular carcinoma. Clin Mol Hepatol. 2012;18(3):330-5.

103. Kumasaka S, Arisaka Y, Tokue A, Higuchi T, Nakajima T, Tsushima Y. A case of multiple hepatic angiomyolipomas with high (18) F-fluorodeoxyglucose uptake. BMC Med Imaging. 2014;14:17.
104. Matsuo Y, Sato M, Shibata T, Morimoto M, Tsuboi K, Shamoto T, et al. Inflammatory pseudotumor of the liver diagnosed as metastatic liver tumor in a patient with a gastrointestinal stromal tumor of the rectum: report of a case. World J Surg Oncol. 2014;12:140.

105. Chong A, Jeong SY, Min JJ. Inflammatory pseudotumours resembling multiple hepatic metastases and their complete regression, as revealed by $18 \mathrm{~F}-\mathrm{FDG}$ PET/CT. Eur J Nucl Med Mol Imaging. 2009;36(7):1199-200.

106. Sharifi S, Murphy M, Loda M, Pinkus GS, Khettry U. Nodular lymphoid lesion of the liver: an immune-mediated disorder mimicking low-grade malignant lymphoma. Am J Surg. 1999;23:302-8.

107. Lin E. Reactive lymphoid hyperplasia of the liver identified by FDG PET. Clin Nucl Med. 2008;33:419-20.

108. Lv A, Liu W, Qian HG, Leng JH, Hao CY. Reactive lymphoid hyperplasia of the liver mimicking hepatocellular carcinoma: incidental finding of two cases. Int J Clin Exp Pathol. 2015;8:5863-9.

109. Tan GJ, Berlangieri SU, Lee ST, Scott AM. FDG-PET/CT in the liver: lesions mimicking malignancies. Abdom Imaging. 2014;39(1):187-95.

110. Vaidyanathan S, Patel CN, Scarsbrook AF, Chowdhury FU. FDG$\mathrm{PET} / \mathrm{CT}$ in infection and inflammation-current and emerging clinical applications. Clin Radiol. 2015;70(7):787-800.

111. Zhuang H, Alavi A. 18-fluorodeoxyglucose positron emission tomographic imaging in the detection and monitoring of infection and inflammation. Semin Nucl Med. 2002;32:47-59.

112. Paik JY, Lee KH, Choe YS, Choi Y, Kim BT. Augmented 18FFDG uptake in activated monocytes occurs during the priming process and involves tyrosine kinases and protein kinase C. J Nucl Med. 2004;45:124-8.

113. Meller J, Sahlmann CO, Scheel AK. 18F-FDG PET and PET/ CT in fever of unknown origin. J Nucl Med. 2007;48(1):35-45.

114. Mochizuki T, Tsukamoto E, Kuge Y, Kanegae K, Zhao S, Hikosaka K, et al. FDG uptake and glucose transporter subtype expressions in experimental tumor and inflammation models. J Nucl Med. 2001;42(10):1551-5.

115. Zhao S, Kuge Y, Tsukamoto E, Mochizuki T, Kato T, Hikosaka $\mathrm{K}$, et al. Fluorodeoxyglucose uptake and glucose transporter expression in experimental inflammatory lesions and malignant tumours: effects of insulin and glucose loading. Nucl Med Commun. 2002;23(6):545-50.

116. Kubota R, Yamada S, Kubota K, Ishiwata K, Tamahashi N, Ido T. Intratumoral distribution of fluorine-18-fluorodeoxyglucose in vivo: high accumulation in macrophages and granulation tissues studied by microautoradiography. J Nucl Med. 1992;33:1972-80.

117. DeLeve LD, Shulman HM, McDonald GB. Toxic injury to hepatic sinusoids: sinusoidal obstruction syndrome (venoocclusive disease). Semin Liver Dis. 2002;22:27-422.

118. Shin NY, Kim MJ, Lim JS, Park MS, Chung YE, Choi JY, et al. Accuracy of gadoxetic acid-enhanced magnetic resonance imaging for the diagnosis of sinusoidal obstruction syndrome in patients with chemotherapy-treated colorectal liver metastases. Eur Radiol. 2012;22(4):864-71.

119. Kawai T, Yamazaki S, Iwama A, Higaki T, Sugitani M, Takayama T. Focal Sinusoidal Obstruction Syndrome Caused by Oxaliplatin-Induced Chemotherapy: A Case Report. Hepat Mon. 2016;16(9):e37572.

120. Choi JH, Won YW, Kim HS, Oh YH, Lim S, Kim HJ. Oxaliplatin-induced sinusoidal obstruction syndrome mimicking metastatic colon cancer in the liver. Oncol Lett. 2016;11(4):2861-4.

Publisher's Note Springer Nature remains neutral with regard to jurisdictional claims in published maps and institutional affiliations. 